\begin{tabular}{|c|c|}
\hline Title & Measurement-Based Performance Evaluation of MIMO Spatial Multiplexing in a Multipath-Rich Indoor Environment \\
\hline Author(s) & Nishimoto, Hiroshi; Ogawa, Y asutaka; Nishimura, Toshihiko; Ohgane, Takeo \\
\hline Citation & $\begin{array}{l}\text { IEEE Transactions on A ntennas and Propagation, 55(12), 3677-3689 } \\
\text { https://doi.org/10.1109/T A P.2007.910303 }\end{array}$ \\
\hline Issue Date & 2007-12 \\
\hline Doc URL & http:/hdl.handle.net/2115/32296 \\
\hline Rights & $\begin{array}{l}\text { C2007 IEEE. Personal use of this material is permitted. However, permission to reprint/republish this material for } \\
\text { advertising or promotional purposes or for creating new collective works for resale or redistribution to servers or lists, } \\
\text { or to reuse any copyrighted component of this work in other works must be obtained from the IEEE. IEEE, IEEE } \\
\text { Transactions on A ntennas and Propagation, 55(12), 2007, p3677-3689 }\end{array}$ \\
\hline Type & article \\
\hline File Information & 04388117.pdf \\
\hline
\end{tabular}

Instructions for use 


\title{
Measurement-Based Performance Evaluation of MIMO Spatial Multiplexing in a Multipath-Rich Indoor Environment
}

\author{
Hiroshi Nishimoto, Student Member, IEEE, Yasutaka Ogawa, Senior Member, IEEE, \\ Toshihiko Nishimura, Member, IEEE, and Takeo Ohgane, Member, IEEE
}

\begin{abstract}
Multiple-input multiple-output (MIMO) spatial multiplexing that needs to separate and detect transmitted signal streams by using processing at the receiver end can increase the data rates of transmissions on independent and identically distributed (i.i.d.) channels. Such channels have been considered to exist in nonline-of-sight (NLOS) environments. However, actual communications may also be conducted in line-of-sight (LOS) environments. While an LOS component can increase the received power level, it may also cause correlated channels that make it difficult to detect the transmitted streams. In this paper, we describe the performance of $4 \times 4$ MIMO spatial multiplexing based on LOS and NLOS channel measurements in an indoor environment. For eight configurations of uniform linear arrays (four antenna spacings and two array orientations), we evaluated the cumulative distribution function (CDF) of the channel capacity and bit error rate performance versus transmit power, and we analyzed them in terms of antenna pattern, fading correlation, CDFs of MIMO channel elements, and CDFs of eigenvalues. Results show that, despite higher fading correlations and non i.i.d. channel characteristics, the performance of MIMO spatial multiplexing in the LOS environment is better than that in the NLOS one. However, the performance in the measured LOS environment largely depends on the MIMO configuration.
\end{abstract}

Index Terms-Array element pattern, bit error rate (BER), channel capacity, channel element distribution, eigenvalue distribution, fading correlation, indoor channel measurement, multiple-input multiple-output (MIMO), mutual coupling, spatial multiplexing.

\section{INTRODUCTION}

$\mathbf{T}$ HE multiple-input multiple-output (MIMO) system in which multiple antennas are placed at both the transmitter and receiver end can potentially meet the growing demand for higher capacity in wireless communications [1]-[5]. One of the applications of the MIMO system is spatial multiplexing in which each transmit antenna sends an independent signal stream with equal power allocation [6]-[8]. When information on the channel state is not available at the transmitter end, this technique seems to be a promising way to increase data transfer speeds without expanding the frequency bandwidth.

Manuscript received September 16, 2005; revised May 15, 2007. This work was supported in part by a Research Fellowship for Young Scientists from the Japan Society for the Promotion of Science.

The authors are with the Graduate School of Information Science and Technology, Hokkaido University, Sapporo 060-0814, Japan (e-mail: hn@ist.hokudai.ac.jp; ogawa@ist.hokudai.ac.jp; nishim@ist.hokudai.ac.jp; ohgane@ist.hokudai.ac.jp).

Digital Object Identifier 10.1109/TAP.2007.910303
Coding and signal processing are key elements to successful implementation of a MIMO system. However, issues related to antennas and electromagnetic propagation also play a significant role in determining MIMO system performance [9]. A low correlation between channels is desirable because spatial multiplexing needs to demultiplex the received signals in order to detect the transmitted streams at the receiver end. Availability of spatial multiplexing has been assessed for independent and identically distributed (i.i.d.) Rayleigh fading channels caused by many scattered signals from surrounding objects. Fades between pairs of transmit-receive antenna elements (channels) are, however, correlated in real propagation environments due to insufficient antenna spacing [10]. Moreover, since mutual coupling effects [11]-[16] exist in a multiple antenna system, characteristics of each antenna vary from those of a single isolated antenna case. Therefore, in actual MIMO communication systems, there is no guarantee that channels are i.i.d. and even that MIMO channel elements obey identically distributed fading.

Uncorrelated channels generally may exist in nonline-of-sight (NLOS) environments where there is no direct wave from the transmitter to the receiver. However, there are many situations in which communications are done also in line-of-sight (LOS) environments. In such cases, while the LOS component can increase the received power level, the channels lose independence and are correlated. Highly correlated channels may make it difficult to detect the transmitted streams. On the other hand, many radio propagation measurement campaigns have already been conducted on MIMO systems [17]-[24]. So far, most of researchers have evaluated the performance of the MIMO systems as a function of average SNR. Because of the evaluation, some reports have presented that NLOS environments give higher channel capacities than LOS ones [17], [22]. However, we must raise the following issue regarding these conclusions. In NLOS environments, the transmit power must be higher than in LOS environments in order to obtain the same SNR. In [17], while channel capacities under the same SNR condition are evaluated for both LOS and NLOS environments, it is also briefly discussed that the comparison is not necessarily fair. Moreover, it has been reported in [23] and [24] that the actual SNR enlarged by the LOS component provides higher channel capacities. The authors agree with their viewpoint and consider that the performance evaluation of the MIMO system should be done under the same transmit power condition.

In measurement-based studies, MIMO systems have ordinarily been evaluated using channel capacities [17]-[24]. Channel capacity is, however, the limit of digital communications given by the information theory. It would be achieved 


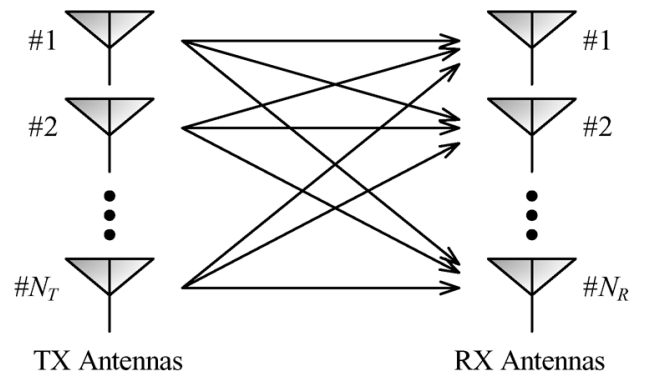

Fig. 1. A model of MIMO spatial multiplexing.

only if we employed an ideal transmission method that includes coding and modulation. From the implementational viewpoint, we consider that not only channel capacity but also bit error rate (BER) can be used to evaluate digital wireless communications.

On the basis of these ideas, we conducted a MIMO channel measurement campaign in an indoor propagation environment where there are many scattered waves. While keeping a constant distance between the transmitter and the receiver, we measured $4 \times 4$ MIMO channels with mutual coupling between antennas in both LOS and NLOS conditions. The performance of MIMO spatial multiplexing in both the environments was examined under the same transmit power condition. In this paper, we used channel capacity for the evaluation of ideal spatial multiplexing, and used BER under a constant bit rate requirement for the case of simple spatial filtering, which proved to be a more realistic and practical performance evaluation.

To investigate the effects of mutual coupling on the measured channel responses, we paid attention to array element patterns. Compared with the single antenna case, the antenna gain with mutual coupling depends on the direction and the array configuration. In particular in LOS environments, the variation of antenna gain changes the impact of the LOS component in the measured channel. We explored, therefore, the influence of array element patterns on the statistical properties of the measured channels and relationship between the influence and the performance of MIMO spatial multiplexing. Moreover, as aforementioned, we examined the BER performance based on the propagation measurement campaign. This means that, MIMO spatial multiplexing is comprehensively evaluated in the range from antennas and propagation to signal processing in this paper.

This paper is organized as follows. Section II defines the model of MIMO spatial multiplexing. Section III outlines our measurement campaign and describes characteristics of the measurement environment. Section IV reports the performance of $4 \times 4$ MIMO systems using the measured data. We draw conclusions from these results in Section V.

\section{MiMO Spatial MultiPleXing}

Fig. 1 shows the model of MIMO spatial multiplexing. We assume that the transmission bandwidth is so narrow that the fading is flat. Moreover, the MIMO system is assumed to have $N_{T}$ transmit (TX) antennas and $N_{R}$ receive (RX) antennas. If independent signal streams are transmitted from the TX antennas, an $N_{R}$-dimensional received signal vector $\boldsymbol{r}(t)$ is expressed as

$$
\boldsymbol{r}(t)=\boldsymbol{H} \boldsymbol{s}(t)+\boldsymbol{n}(t)
$$

where $\boldsymbol{s}(t)$ is an $N_{T}$-dimensional transmitted signal vector, and $\boldsymbol{n}(t)$ is an $N_{R}$-dimensional additive white Gaussian noise vector. An $N_{R} \times N_{T}$ MIMO channel matrix $\boldsymbol{H}$ consists of channel responses between TX and RX antennas. These are represented as follows:

$$
\begin{aligned}
\boldsymbol{s}(t) & =\left[s_{1}(t), s_{2}(t), \ldots, s_{N_{T}}(t)\right]^{T} \\
\boldsymbol{n}(t) & =\left[n_{1}(t), n_{2}(t), \ldots, n_{N_{R}}(t)\right]^{T} \\
\boldsymbol{H} & =\left[\begin{array}{cccc}
h_{11} & h_{12} & \cdots & h_{1 N_{T}} \\
h_{21} & h_{22} & \cdots & h_{2 N_{T}} \\
\vdots & \vdots & h_{i j} & \vdots \\
h_{N_{R}} & h_{N_{R} 2} & \cdots & h_{N_{R} N_{T}}
\end{array}\right] .
\end{aligned}
$$

Here, the superscript ${ }^{T}$ indicates a transpose. Also, $h_{i j}$, which is an element of the $i$ th row and the $j$ th column in the matrix $\boldsymbol{H}$, denotes a channel from the $j$ th TX antenna to the $i$ th RX antenna.

As will be stated next, the measured MIMO channels have mutual coupling effects. When mutual coupling matrices in the TX and RX arrays are defined as $\boldsymbol{C}_{\mathrm{TX}}$ and $\boldsymbol{C}_{\mathrm{RX}}$, respectively, the measured channel matrix $\boldsymbol{H}$ is expressed as

$$
\boldsymbol{H}=\boldsymbol{C}_{\mathrm{RX}} \overline{\boldsymbol{H}} \boldsymbol{C}_{\mathrm{TX}}^{T}
$$

where $\overline{\boldsymbol{H}}$ is an $N_{R} \times N_{T}$ real channel matrix which does not include mutual coupling effects. When all of $N$ antennas in an $N$-element array are assumed to have the same load impedance $Z_{L}$, an $N \times N$ mutual coupling matrix $C$ is defined as

$$
\boldsymbol{C}^{-1}=\frac{1}{Z_{L}}\left[\begin{array}{cccc}
Z_{L}+Z_{11} & Z_{12} & \cdots & Z_{1 N} \\
Z_{21} & Z_{L}+Z_{22} & \cdots & Z_{2 N} \\
\vdots & \vdots & \ddots & \vdots \\
Z_{N 1} & Z_{N 2} & \cdots & Z_{L}+Z_{N N}
\end{array}\right]
$$

where $Z_{i j}$ is self/mutual impedance between the $i$ th and $j$ th antennas [11], [12]. Note that $\boldsymbol{C}_{\mathrm{TX}}$ and $\boldsymbol{C}_{\mathrm{RX}}$ can actually have different definitions. In this paper, however, we do not discuss them because it is beyond the scope of this paper. We obtained MIMO transfer matrices including mutual coupling effects from channel data measured in actual indoor propagation environments (see later). Using the measured channels, the characteristics and the performance of spatial multiplexing were evaluated.

\section{Mimo Channel Measurement Setup}

The measurement campaign was carried out in a conference room in a building of the Graduate School of Information Science and Technology at Hokkaido University (Fig. 2). The room had many scatterers. The walls consisted of plasterboard around reinforced concrete pillars and metal doors. In this room, we set up TX and RX tables and a vector network analyzer (VNA) 


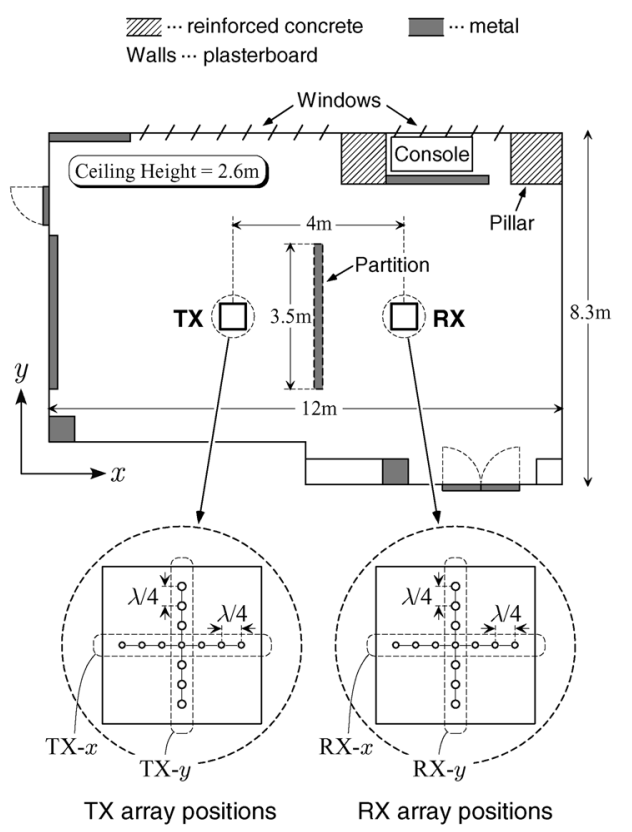

(a)

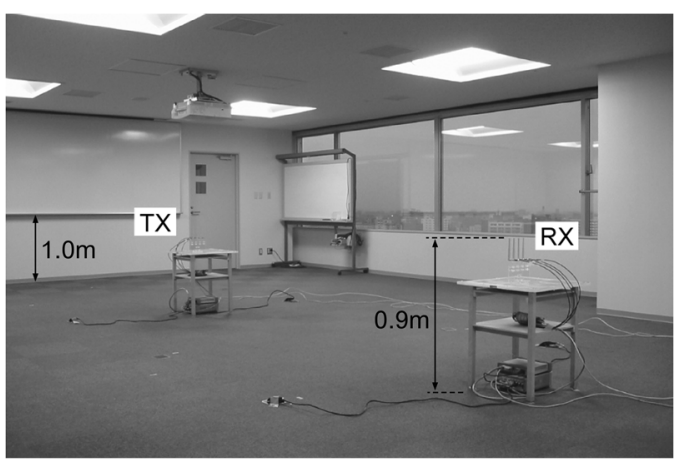

(b)

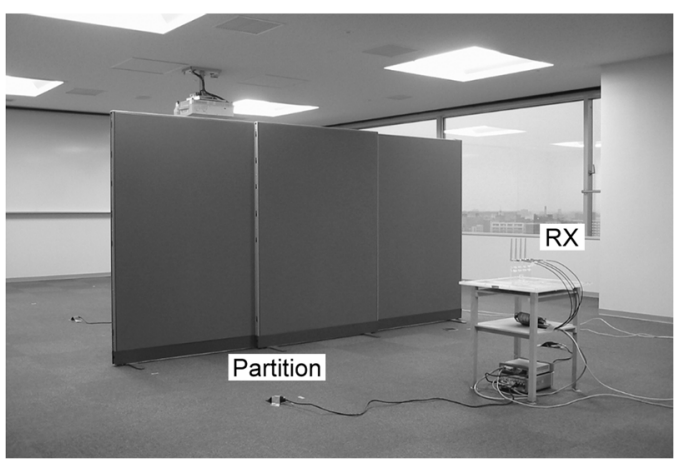

(c)

Fig. 2. Measurement site. (a) Top view. (b) LOS. (c) NLOS.

to measure the channel responses. The measurement band was from 5.15 to $5.4 \mathrm{GHz}$ ( $250 \mathrm{MHz}$ bandwidth), and it was swept with a $156.25 \mathrm{kHz}$ interval (1 601 frequency sample points). Each channel was averaged over 10 snapshots in order to reduce thermal noise included in raw measurements. The TX and RX tables were separated by $4 \mathrm{~m}$, as shown in the dashed circles in Fig. 2(a). The LOS condition was taken as the absence of an obstructing object between the TX and RX tables [Fig. 2(b)]. The NLOS condition was created by placing a metal partition between the TX and RX [Fig. 2(c)]. The partition was large enough

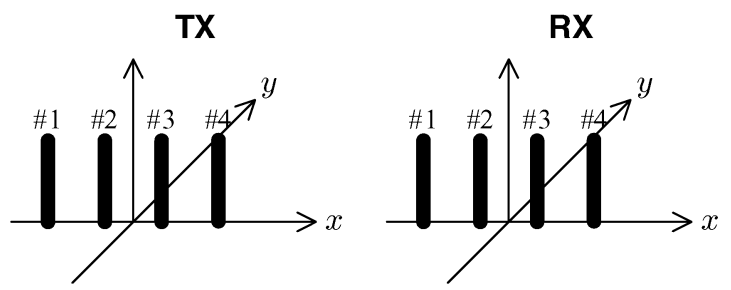

(a)
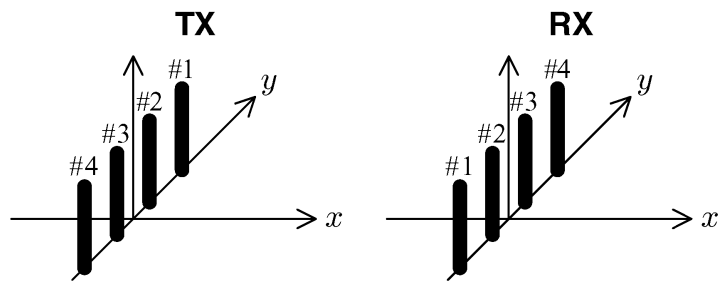

(b)

Fig. 3. Antenna array orientations. (a) TX-x/RX-x. (b) TX-y/RX-y

to avoid deterministic waves, which were diffracted at edges of the partition, arriving at the receiver. ${ }^{1}$ There was a metal screen (whiteboard) on the wall behind the TX table. The height of its bottom was $1 \mathrm{~m}$, and antenna height was $0.9 \mathrm{~m}$ as illustrated in Fig. 2(b). Channel data were obtained while no one was in the room, to ensure statistical stationarity of propagation.

The $x$ and $y$ axes were defined as in Fig. 2(a). The MIMO measurement campaign used uniform linear antenna arrays. TX and $\mathrm{RX}$ antennas aligned along the $x$ axis are denoted as the TX- $x / \mathrm{RX}-x$ orientation [Fig. 3(a)], and antennas aligned along the $y$ axis are denoted as the TX-y/RX- $y$ orientation [Fig. 3(b)]. To obtain spatially different fading channels, there were seven positions on the TX and RX tables for the antenna array mount along the $x$ and $y$ axes separated with an interval of $\lambda / 4(1.5$ $\mathrm{cm}$ ), as shown in the dashed circles in Fig. 2(a), where $\lambda$ denotes the wavelength at $5 \mathrm{GHz}(6 \mathrm{~cm})$. The antenna array orientation corresponds to the array position direction also as shown in the dashed circles in Fig. 2(a). For example, the positions of the TX and the RX arrays for the TX- $x / \mathrm{RX}-x$ orientation were changed in the $x$ direction. The array's antenna spacing (AS) had four values: $0.25 \lambda, 0.50 \lambda, 0.75 \lambda$, and $1.00 \lambda$. By changing the $\mathrm{TX}$ and $\mathrm{RX}$ array positions, we obtained $7 \times 7=49$ spatially different data. Because we had 1601 frequency-domain data, a total of $49 \times 1601=78449$ different MIMO channel matrices were obtained for each array orientation, antenna spacing, and LOS/NLOS condition. All of the characteristics presented in the next section were derived from statistical processing of all the 78449 MIMO channel data.

We employed colinear antennas AT-CL010 (TSS JAPAN Co., Ltd.) designed for omnidirectional characteristics on the horizontal $(x-y)$ plane. All of the antennas had return loss less than $-10 \mathrm{~dB}$ from 5.15 to $5.4 \mathrm{GHz}$. When a single-input singleoutput (SISO) channel was measured in an anechoic chamber (AEC) by using the antennas as the TX and RX ones, the maximum amplitude variation of the observed direct wave was 0.7 $\mathrm{dB}$ in this measurement band. Fig. 4 shows an example of a

${ }^{1}$ The large partition placed for the NLOS condition may have obstructed not only the LOS component but also major scattered waves, as will be shown in Fig. 5 . 


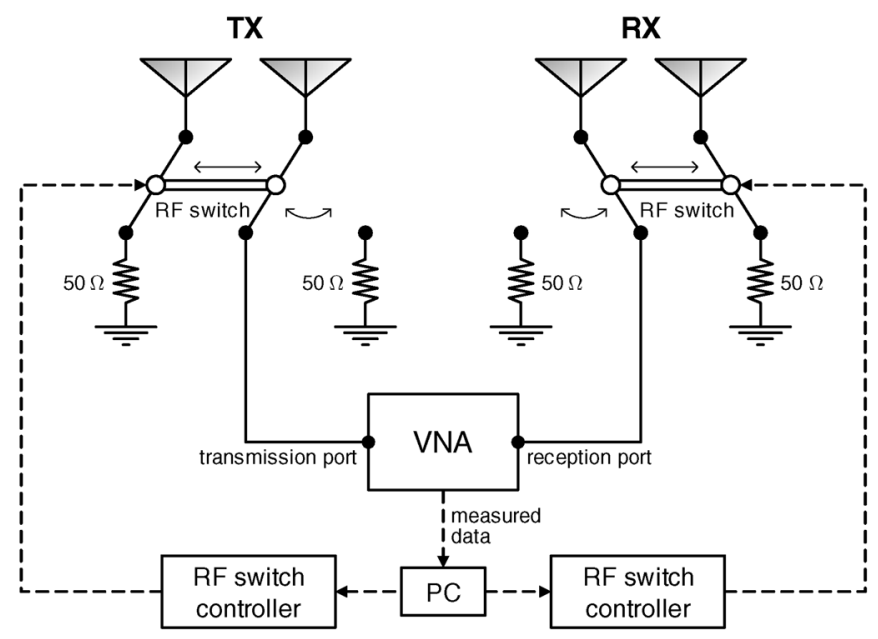

Fig. 4. MIMO channel measurement system.

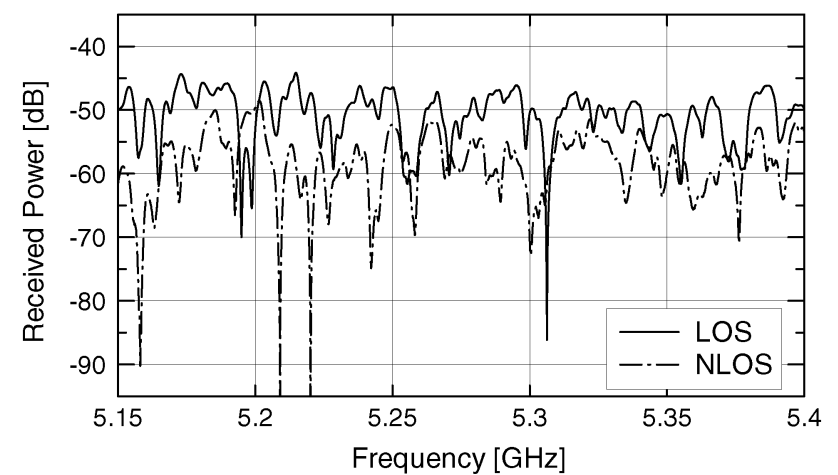

(a)

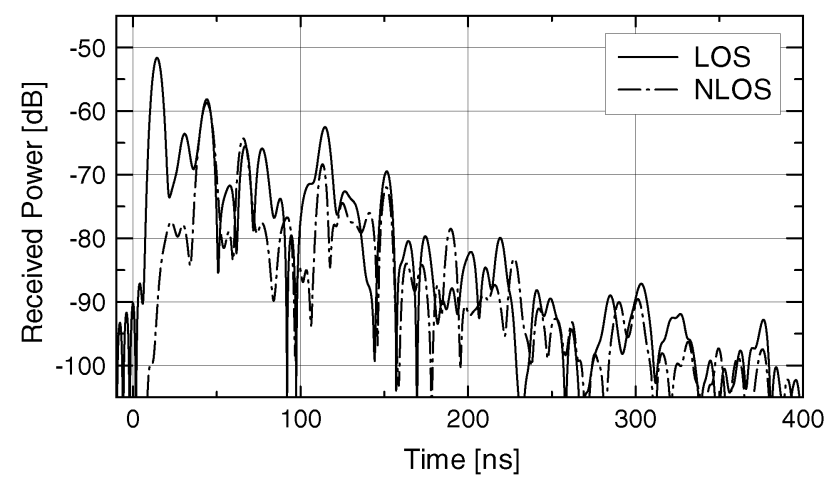

(b)

Fig. 5. Example of measurement data (SISO case). (a) Frequency domain. (b) Time domain.

$2 \times 2$ MIMO measurement system, for which the basic idea is the same for the $4 \times 4$ MIMO system. RF switches at the TX and RX sides were used to select the TX antenna and the RX antenna. According to this selection, we chose each element in the $4 \times 4$ MIMO matrix. We normalized the measured channel responses to calibration data that had been obtained when the cables to the antenna ports from the RF switches were directly connected. Therefore, the calibrated data did not have the frequency characteristics of the cables and switches. The unselected antennas were automatically connected to $50 \Omega$ dummy loads.

Fig. 5 shows example measurements. These data were obtained at the central positions on the TX and RX tables as shown

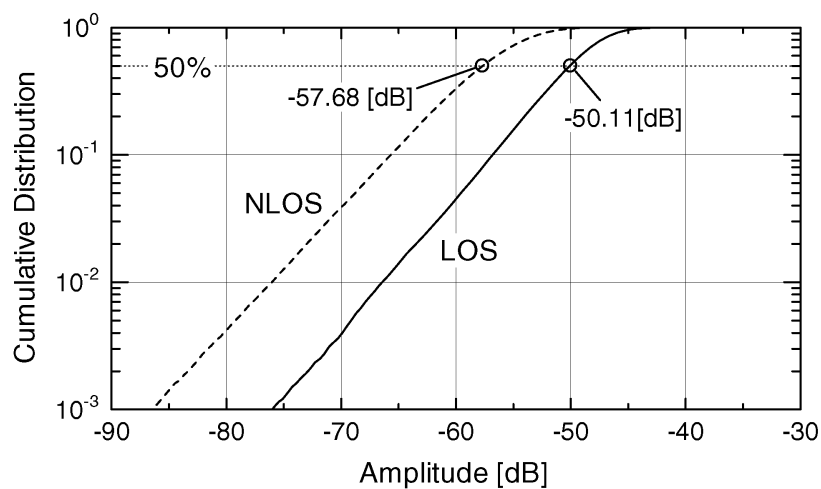

Fig. 6. CDFs of amplitudes (SISO case).

in the dashed circles in Fig. 2(a) when TX and RX ends each used a single antenna (SISO case). Thus, the antennas did not have mutual coupling. Fig. 5(a) shows measured frequency-domain data. It is clear that the received power for the LOS condition was generally larger than the power for the NLOS one due to the direct wave. Time-domain data shown in Fig. 5(b) are the results of performing the IFFT on the frequency-domain data shown in Fig. 5(a). The many peaks for the LOS and NLOS conditions indicate that the measurement environments had many scattered waves. The maximum peak was around $14 \mathrm{~ns}$ for the LOS condition. This is considered to be from the direct wave because the distance of $4 \mathrm{~m}$ between the TX and RX ends gives a propagation time of $4 /\left(3.0 \times 10^{8}\right)=13.3 \mathrm{~ns}$ and the peak disappeared for the NLOS condition. Furthermore, unlike NLOS, LOS gave larger amplitudes for the waves with short propagation delays within $60 \mathrm{~ns}$.

We measured 82 spatially different SISO channels in the frequency band from 5.15 to $5.40 \mathrm{GHz}$ in the LOS and NLOS conditions keeping the distance of $4 \mathrm{~m}$ between the TX and RX tables. The measured SISO channels provided the cumulative distribution function (CDF) of the wave amplitudes as shown in Fig. 6. The received amplitudes for the LOS condition were about $7.6 \mathrm{~dB}$ higher at the $50 \%$ level compared with those for the NLOS condition. The mean received power averaged over space and frequency samples in the SISO-LOS measurement was $G_{\mathrm{LOS}}=-49.26 \mathrm{~dB}\left(1.186 \times 10^{-5}\right)$, and that in the SISO-NLOS one was $G_{\mathrm{NLOS}}=-56.05 \mathrm{~dB}$. Comparing the mean received powers, $G_{\text {LOS }}$ was about $6.8 \mathrm{~dB}$ higher than $G_{\text {NLOS }}$. Also, the mean received power $G_{\text {AEC }}$ was obtained under the SISO and LOS condition in the AEC in which the TX and RX tables were set $4 \mathrm{~m}$ apart. The data were averaged over the space and frequency samples. $G_{\mathrm{AEC}}$ which is the estimated direct wave power was $-51.52 \mathrm{~dB}\left(7.047 \times 10^{-6}\right)$. Since $G_{\mathrm{LOS}}$ is composed of the direct and scattered wave power, the Ricean $K$-factor in the LOS environment can be easily estimated by the following calculation:

$$
\begin{aligned}
K & =\frac{G_{\mathrm{AEC}}}{G_{\mathrm{LOS}}-G_{\mathrm{AEC}}} \\
& =\frac{7.047 \times 10^{-6}}{1.186 \times 10^{-5}-7.047 \times 10^{-6}} \\
& =1.66 \mathrm{~dB} .
\end{aligned}
$$

The scattered wave power was, therefore, comparable to the direct wave power. Moreover, the scattered wave power in the 
LOS condition $\left(G_{\mathrm{LOS}}-G_{\mathrm{AEC}}=-53.18 \mathrm{~dB}\right)$ was $2.87 \mathrm{~dB}$ higher than $G_{\text {NLOS }}$. It is natural that an LOS component causes higher received power. However, this result indicates that, under the LOS condition of this room, the direct wave also increased scattered signal power.

The channel response $h$ and MIMO channel matrix $\boldsymbol{H}$ used in the next section are given by the following normalizations of the measured $\hat{h}$ and $\hat{\boldsymbol{H}}$

$$
\begin{gathered}
h=\frac{\hat{h}}{\sqrt{G_{\mathrm{AEC}}}} \\
\boldsymbol{H}=\frac{\hat{\boldsymbol{H}}}{\sqrt{G_{\mathrm{AEC}}}} .
\end{gathered}
$$

We can say that the channel response is normalized to the direct wave amplitude.

\section{Performance of $4 \times 4$ Mimo Systems}

\section{A. Antenna Patterns}

When multiple antennas are closely arrayed, they have mutual coupling and their antenna patterns change. A MIMO system has antenna arrays at both ends, so we cannot ignore the effect of the changing pattern on the MIMO performance. Thus, before presenting the measured characteristics of the MIMO channels, we examine the antenna patterns for each four-element linear array.

Fig. 7 shows the patterns for each four-element uniform linear array treated in this measurement campaign (solid curves). Azimuth patterns for $\mathrm{AS}=0.25 \lambda, 0.50 \lambda, 0.75 \lambda$, and $1.00 \lambda$ are shown in Fig. 7(a)-(d), respectively. For comparison, the pattern of a single antenna is shown with a dashed curve. We see that the single antenna has an almost omnidirectional pattern when it does not have the mutual coupling effect. In the multiple antenna case, however, the patterns are significantly different from an omnidirectional one. The antenna gain decreases as the AS becomes smaller. On the other hand, the patterns tend to become similar to the omnidirectional one as the AS becomes larger. The numbers under each pattern correspond to the ones in Fig. 3. Given the TX- $x / \mathrm{RX}-x$ orientation, the RX end is located in the $0^{\circ}$ direction with respect to the TX end, and the TX end is located in the $180^{\circ}$ direction with respect to the RX end. Thus, the direct wave departs from the TX end in the $0^{\circ}$ direction, and arrives at the RX end in the $180^{\circ}$ direction. On the other hand, given the TX- $y / \mathrm{RX}-y$ orientation, the RX end is located in the $90^{\circ}$ direction with respect to the TX end, and the TX end is also located in the $90^{\circ}$ direction with respect to the RX end. Thus, the direct wave departs from the TX end and arrives at the $\mathrm{RX}$ end in the $90^{\circ}$ direction. As for the $0^{\circ}$ and $180^{\circ}$ directions, the gain tends to be small. On the other hand, especially in the cases of $\mathrm{AS}=0.50 \lambda$ and $0.75 \lambda$, the gain in the $90^{\circ}$ direction is higher than in the single antenna case.

\section{B. Fading Correlations}

To investigate the characteristics of the MIMO channels measured in the propagation environment, we examined fading correlations [18]. The RX fading correlation $\rho_{\mathrm{RX}, i j}$ between the $i$ th and $j$ th RX antennas and the TX fading correlation $\rho_{\mathrm{TX}, i j}$ between the $i$ th and $j$ th TX antennas are given by (12) and (13) shown at the bottom of the next page. Here, $k$ and $l$ indicate the TX antenna number and RX antenna number, respectively. $m$ represents the number of MIMO channel data and $M$ is the number of total data (78 449). The absolute values of the fading correlations are in the range from 0 to 1 . In addition, the correlations $\left|\rho_{\mathrm{RX}, i j}\right|$ and $\left|\rho_{\mathrm{TX}, i j}\right|(i \neq j)$ have ${ }_{N_{R}} C_{2}$ and ${ }_{N_{T}} C_{2}$ different values, respectively, where an operator ${ }_{p} C_{q}$ denotes the total number of combinations of $q$-subsets possible out of a set of $p$ distinct items. That is, ${ }_{p} C_{q}=p ! /\{q !(p-q) !\}$ holds.

Using (12) and (13), we obtained the RX and TX fading correlations for the measured $4 \times 4$ MIMO channels. Since all of the MIMO cases have almost the same correlation values between the RX and TX ones, we will omit discussion on the TX correlations and discuss only the RX fading correlations $\left|\rho_{\mathrm{RX}, i j}\right|$. The correlations are drawn in the three-dimensional style as shown in Fig. 8 because the combination of the RX antennas gives ${ }_{4} C_{2}=6$ values. This plot is in the same style as in [18]. Fig. 8(a) and (b) show the correlations in the NLOS condition, and Fig. 8(c) and (d) show them in the LOS condition. In addition, Fig. 8(a) and (c) are for the TX- $x / \mathrm{RX}-x$ orientation, and Fig. 8(b) and (d) are for the TX- $y / R X-y$ one. In the NLOS condition, the fading correlations generally have low values, and they become lower as AS increases. Higher correlations in the LOS condition are due to the LOS component that is a deterministic signal.

Focusing on the correlations in the LOS condition, we find that the TX- $y / \mathrm{RX}-y$ orientation tends to provide higher correlations than the TX- $x / \mathrm{RX}-x$ one. We can consider two reasons for this. The direct wave and reflected waves from the walls behind the TX and behind the RX were conjectured to be dominant. These reflected rays along the $x$ axis lowered the correlation for the TX- $x / \mathrm{RX}-x$ orientation, but did not cause the decorrelation for the TX- $y / \mathrm{RX}-y$ case. We can analyze the other reason by considering the antenna patterns shown in Fig. 7. Narrow spacing seems to give high correlations for both of the orientations in the case of AS $=0.25 \lambda$. However, as mentioned in Section IV-A, the cases of AS $=0.50 \lambda$ and $0.75 \lambda$ give higher gain in the $90^{\circ}$ direction, which corresponds to the direct path.

$$
\begin{aligned}
\rho_{\mathrm{RX}, i j} & =\frac{\sum_{m=1}^{M} \sum_{k=1}^{N_{T}} h_{i k}^{*}(m) h_{j k}(m)}{\sqrt{\sum_{m=1}^{M} \sum_{k=1}^{N_{T}}\left|h_{i k}(m)\right|^{2}} \sqrt{\sum_{m=1}^{M} \sum_{k=1}^{N_{T}}\left|h_{j k}(m)\right|^{2}}} \\
\rho_{\mathrm{TX}, i j} & =\frac{\sum_{m=1}^{M} \sum_{l=1}^{N_{R}} h_{l i}^{*}(m) h_{l j}(m)}{\sqrt{\sum_{m=1}^{M} \sum_{l=1}^{N_{R}}\left|h_{l i}(m)\right|^{2}} \sqrt{\sum_{m=1}^{M} \sum_{l=1}^{N_{R}}\left|h_{l j}(m)\right|^{2}}}
\end{aligned}
$$



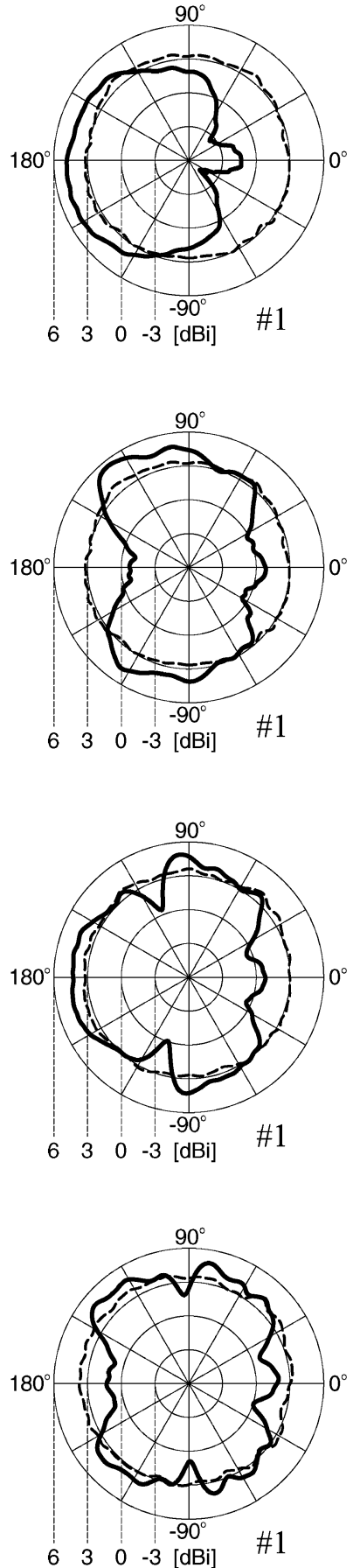
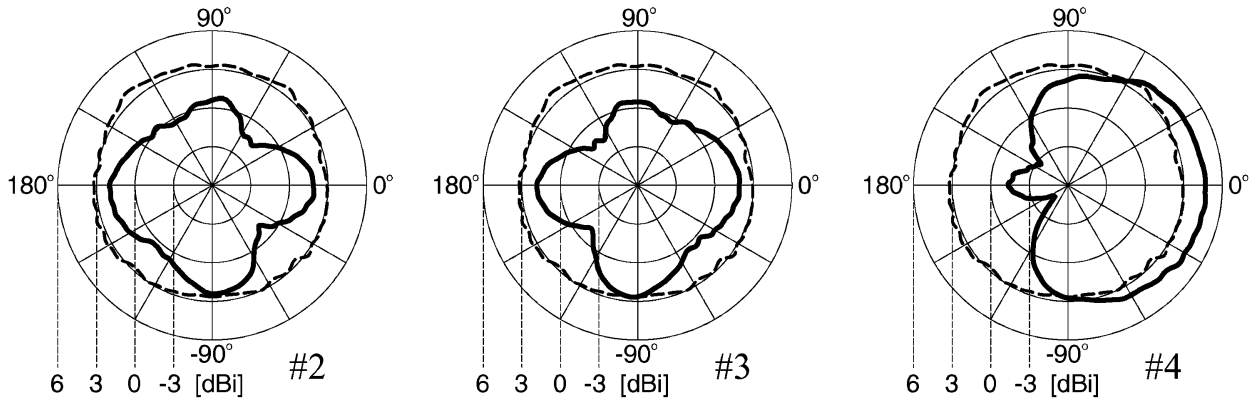

(a)
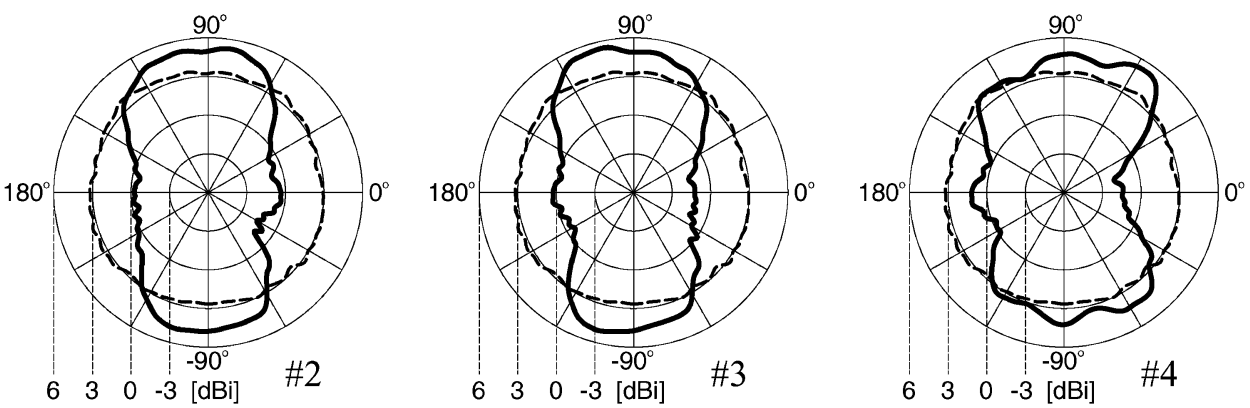

(b)
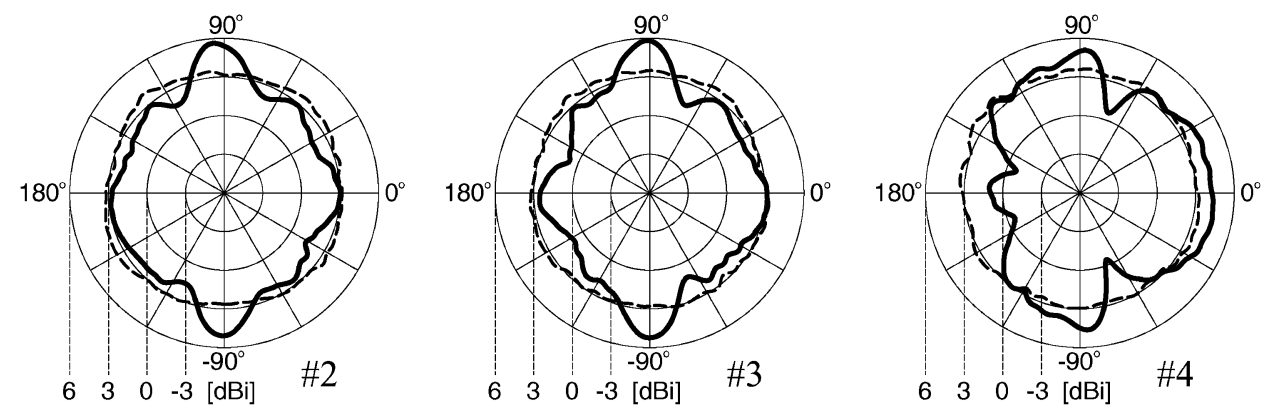

(c)
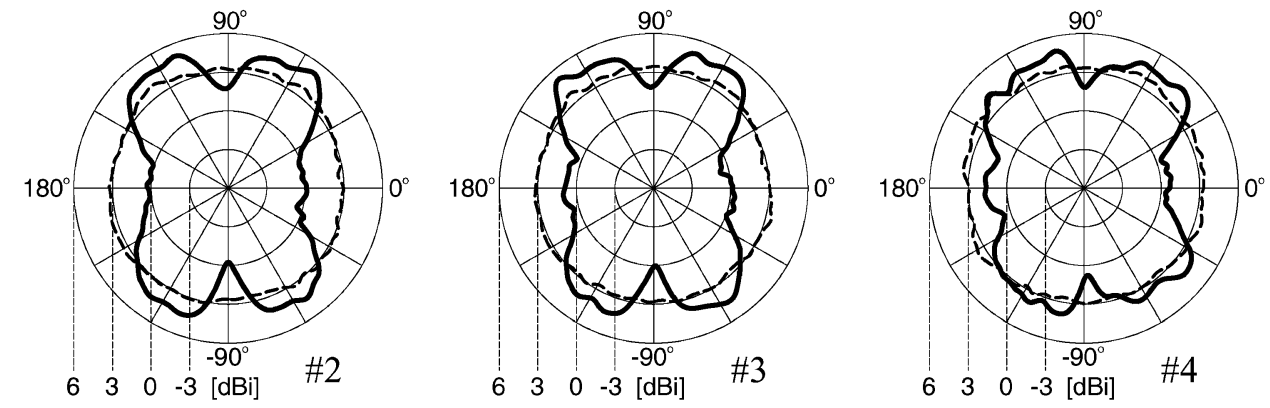

(d)

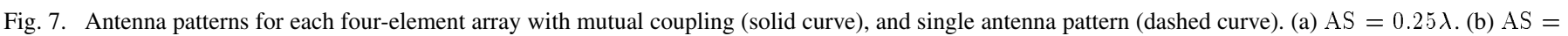
$0.50 \lambda$. (c) $\mathrm{AS}=0.75 \lambda$. (d) $\mathrm{AS}=1.00 \lambda$.

Consequently, the effect of the direct wave becomes stronger, so that we have higher correlations for the TX- $y / \mathrm{RX}-y$ orientation. The patterns for AS $=1.00 \lambda$ incidentally show a little dip in the antenna pattern in the $90^{\circ}$ direction. This decreases the effect of the direct wave and causes lower correlation values in the TX- $y / \mathrm{RX}-y$ orientation.

\section{CDFs of Channel Elements in MIMO Matrices}

Many MIMO channel models assume that each element in a channel matrix obeys i.i.d. fading. However, actual MIMO systems can be strongly affected by propagation environments and mutual coupling between antennas. Actual channel elements, thereby, may have different statistical characteristics. As stated in the previous subsection, some LOS channels have very high correlations, and are never independent. Here, we attempt to determine whether the indoor MIMO channels are identical or not. Using the measured 78449 MIMO channels, we examined CDFs of amplitudes of each channel element as shown in Fig. 9. Data for the TX- $x / \mathrm{RX}-x$ orientation are shown in Fig. 9(a)-(d), and data for the TX-y/RX-y 


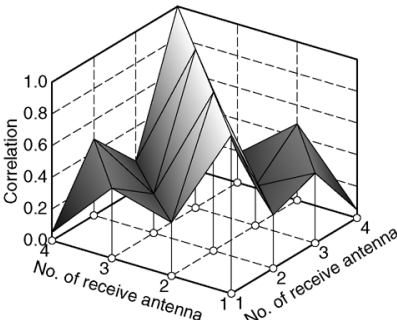

$\mathrm{AS}=0.25 \lambda$

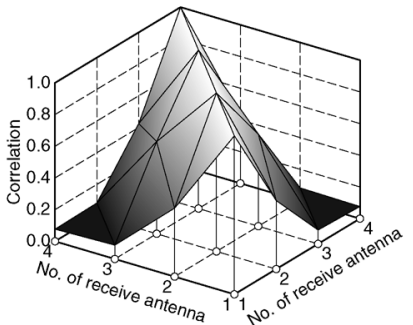

$\mathrm{AS}=0.25 \lambda$

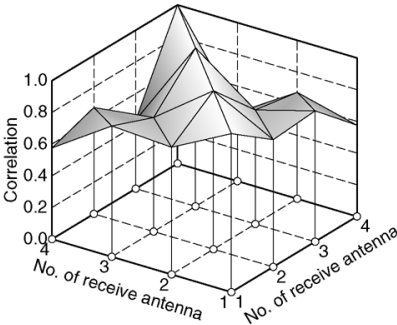

$\mathrm{AS}=0.25 \lambda$

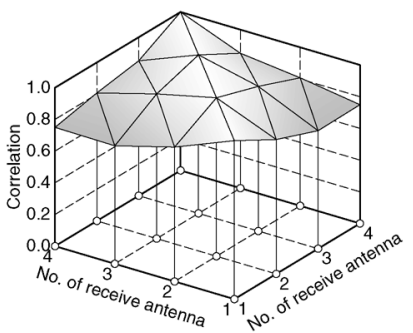

$\mathrm{AS}=0.25 \lambda$

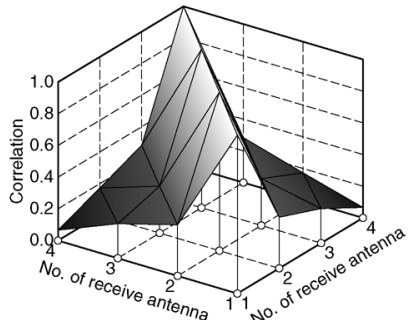

$\mathrm{AS}=0.50 \lambda$

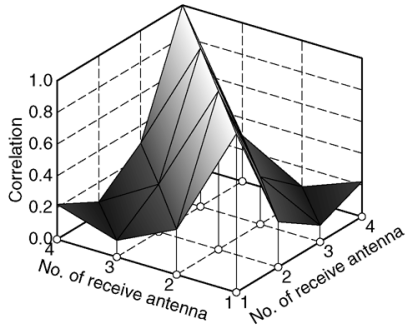

$\mathrm{AS}=0.50 \lambda$

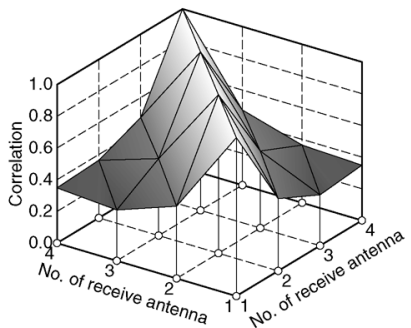

$\mathrm{AS}=0.50 \lambda$

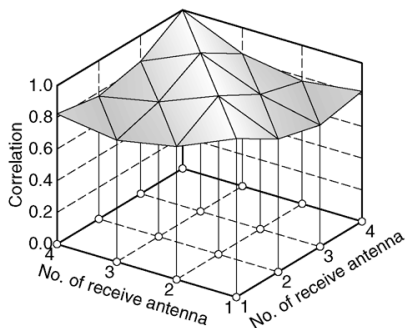

$\mathrm{AS}=0.50 \lambda$

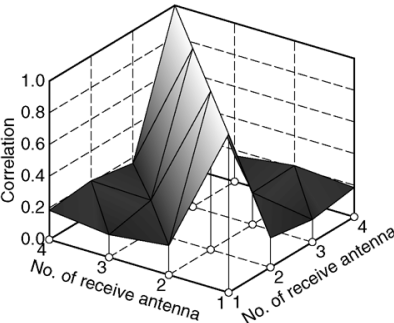

$\mathrm{AS}=0.75 \lambda$

(a)

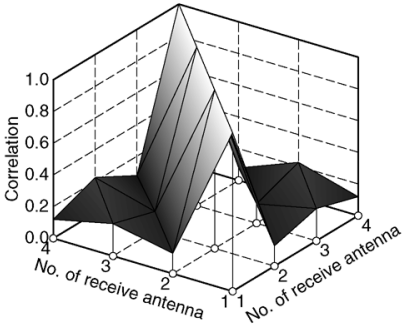

$\mathrm{AS}=0.75 \lambda$

(b)

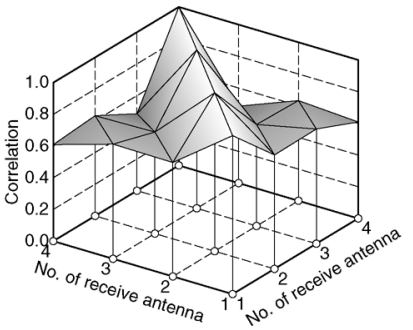

$\mathrm{AS}=0.75 \lambda$

(c)

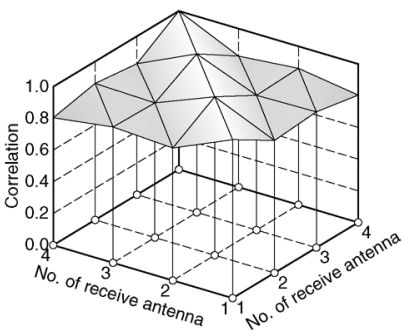

$\mathrm{AS}=0.75 \lambda$

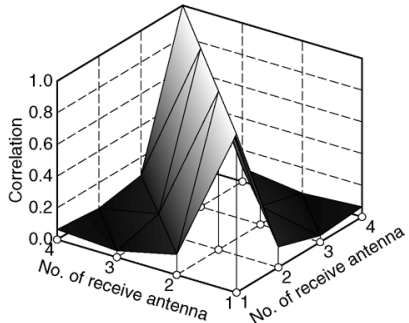

$\mathrm{AS}=1.00 \lambda$

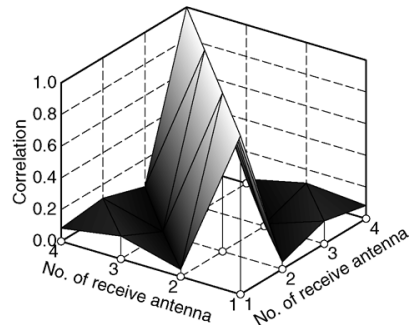

$\mathrm{AS}=1.00 \lambda$

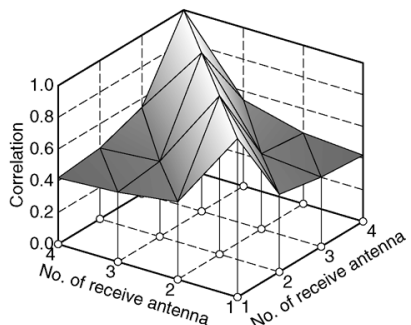

$\mathrm{AS}=1.00 \lambda$

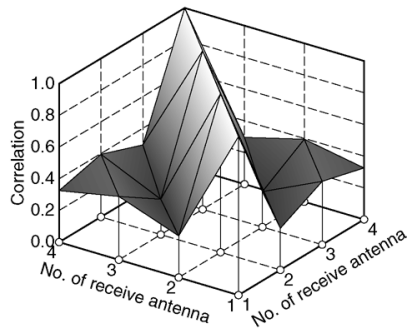

$\mathrm{AS}=1.00 \lambda$

(d)

Fig. 8. RX fading correlations $\left|\rho_{\mathrm{RX}, \mathrm{ij}}\right|$ for the measured $4 \times 4$ MIMO channels. (a) NLOS, TX- $x / \mathrm{RX}-x$. (b) NLOS, TX-y/RX-y. (c) LOS, TX- $x / \mathrm{RX}-x$. (d) LOS, TX- $y /$ RX- $y$.

orientation are shown in Fig. 9(e)-(h). In addition, pairs of Fig. 9(a) and (e), (b) and (f), (c) and (g), and (d) and (h) show the CDFs for the cases of $\mathrm{AS}=0.25 \lambda, 0.50 \lambda, 0.75 \lambda$, and $1.00 \lambda$, respectively. Each of the LOS/NLOS condition in the graph has $4 \times 4=16 \mathrm{CDF}$ curves $\left(\left|h_{11}\right|,\left|h_{12}\right|,\left|h_{13}\right|, \ldots,\left|h_{44}\right|\right.$. The abscissa value is different from that in Fig. 6 owing to the normalization by (11). Also, for each case in the LOS condition, the average Ricean factor $K_{\text {avg }}$ estimated from all the $16 \mathrm{CDFs}$ of channel elements is put on each graph. First, as in Fig. 6, the MIMO channel elements in the LOS condition generally have higher amplitudes than those in the NLOS one. Second, except for the narrowest spacing cases of AS $=0.25 \lambda$, distributions under the NLOS condition are independent of the antenna spacing and array orientation, and differ less than about $2 \mathrm{~dB}$. Looking at the region where the cumulative frequencies are less than the $10 \%$ level and the curves are almost straight lines, we can see that all the CDFs increase by almost an order of magnitude with an amplitude increment of $10 \mathrm{~dB}$. Thus, the NLOS channels are Rayleigh fading channels. In the previous subsection, we verified that almost all of the cases under the NLOS condition give low fading correlations (Fig. 8). Hence, we can say that MIMO channels under the NLOS condition with AS equal to or greater than $0.50 \lambda$ obey almost i.i.d. Rayleigh fading. As for the cases of $\mathrm{AS}=0.25 \lambda$, the difference at the $10 \%$ level is a maximum of approximately $3 \mathrm{~dB}$. The authors consider that it was caused 


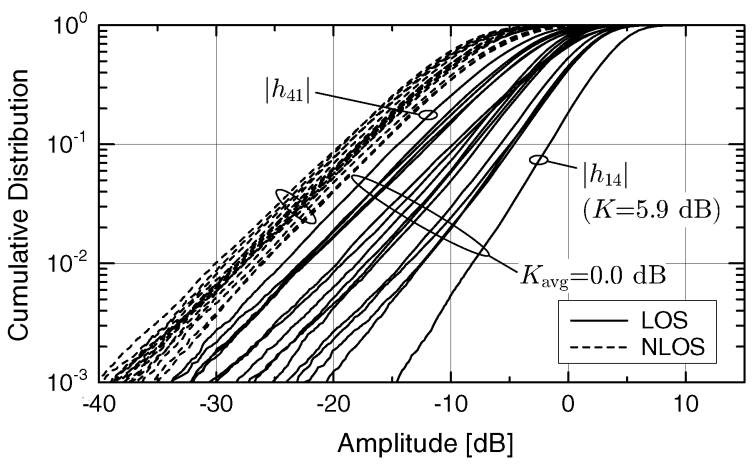

(a)

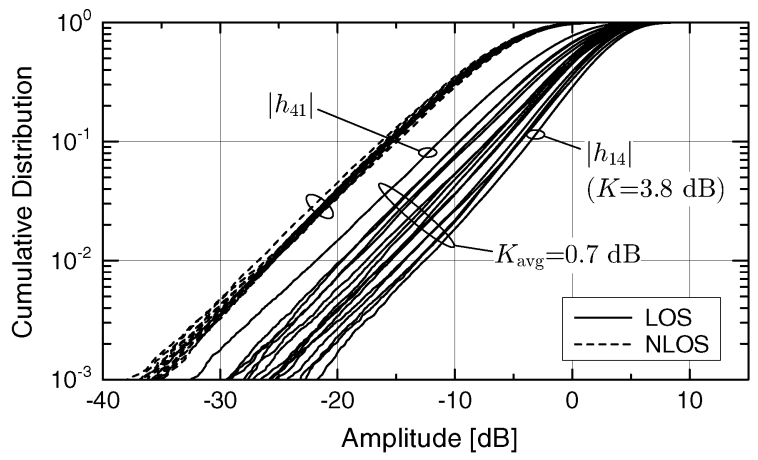

(c)

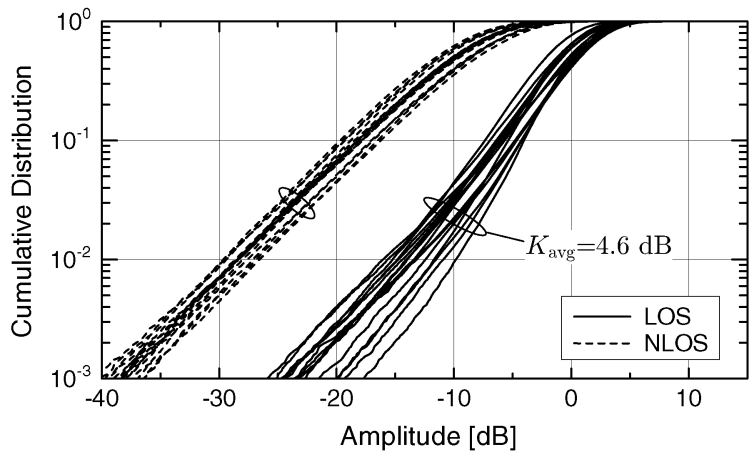

(e)

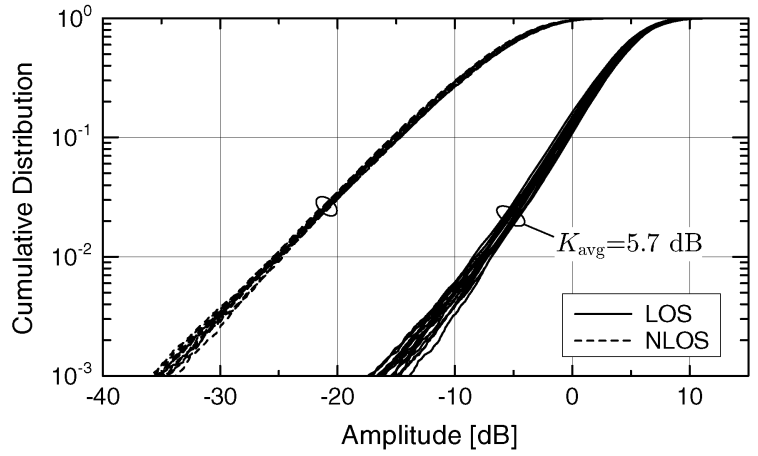

(g)

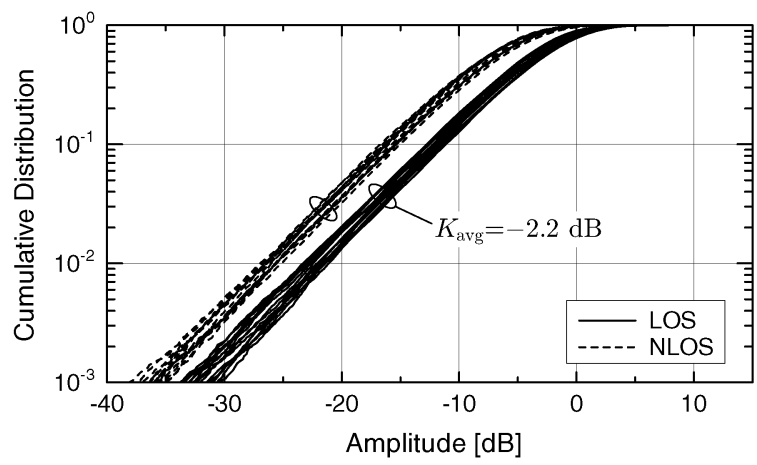

(b)

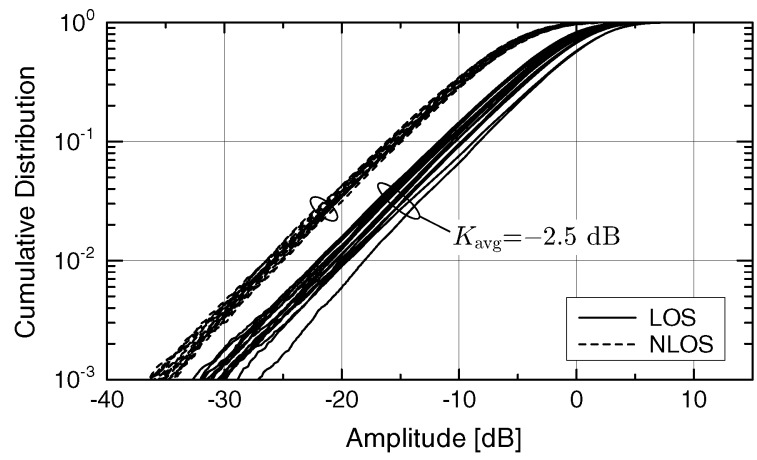

(d)

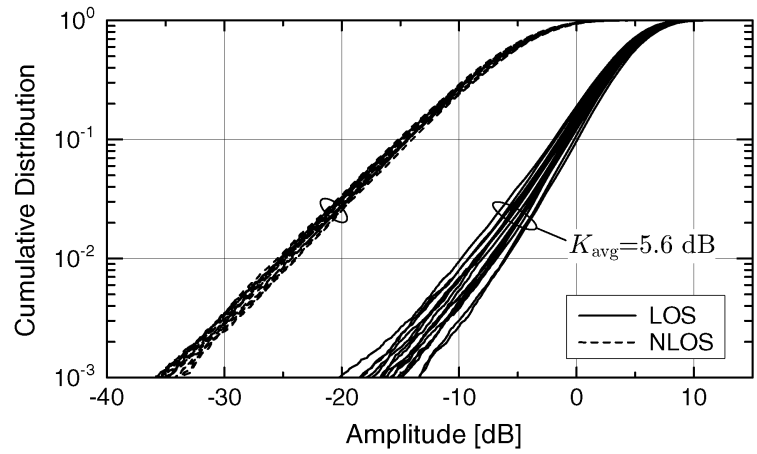

(f)

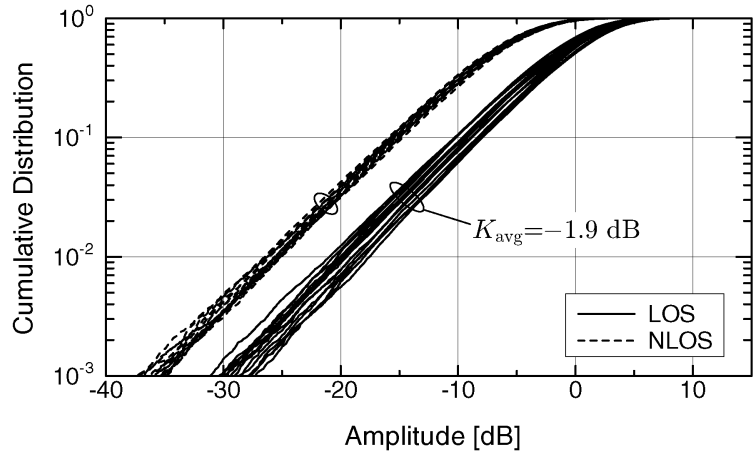

(h)

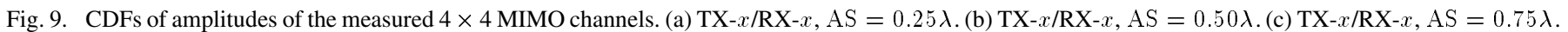
(d) $\mathrm{TX}-x / \mathrm{RX}-x, \mathrm{AS}=1.00 \lambda$. (e) TX- $y / \mathrm{RX}-y, \mathrm{AS}=0.25 \lambda$. (f) TX-y/RX- $y, \mathrm{AS}=0.50 \lambda$. (g) TX- $y / \mathrm{RX}-y, \mathrm{AS}=0.75 \lambda$. (h) TX-y/RX- $y$, AS $=1.00 \lambda$.

by different antenna gain among antenna elements, especially significant gain loss in inner two elements \#2 and \#3, due to mutual coupling effects as shown in Fig. 7(a).

However, channels under the LOS condition do not behave in this way. The amplitudes and gradients of the CDFs differ depending on the antenna spacing and array orientation. Since the LOS condition has the direct wave, the antenna gain has a great influence on the channel distributions. We can explain this behavior by using the antenna patterns shown in Fig. 7. When the gain in the direct wave's direction is high, CDFs become lo- 
cated in a higher amplitude region and their gradients become steeper. That is, the fading is Ricean with a large $K$-factor. On the other hand, when the gain toward the direct wave is low, the amplitudes are distributed in a lower region. Consider the cases of $\mathrm{AS}=0.25 \lambda$ and $0.75 \lambda$ in the TX- $x / \mathrm{RX}-x$ orientation. From Fig. 9(a) and (c), we see that the channel distributions are significantly different. In these graphs, the CDF of $\left|h_{14}\right|$ is in the highest amplitude region and has the steepest gradient (largest $K$-factor) among the MIMO channel elements. For example, in the case of $\mathrm{AS}=0.25 \lambda$, while $K_{\mathrm{avg}}$ is $0.0 \mathrm{~dB}$, the $K$-factor of $\left|h_{14}\right|$ is $5.9 \mathrm{~dB}$. In the case of $\mathrm{AS}=0.75 \lambda, K_{\mathrm{avg}}$ is $0.7 \mathrm{~dB}$ whereas the $K$-factor of $\left|h_{14}\right|$ is $3.8 \mathrm{~dB}$. On the other hand, the CDF of $\left|h_{41}\right|$ is in the lowest region under the LOS condition. Note that each antenna index corresponds to the one shown in Figs. 3 and 7. Here, let us consider the channels and antenna patterns shown in Fig. 7. As for channel $h_{14}$ in the TX- $x$ /RX- $x$ orientation, the direct wave departs from TX antenna \#4 in the $0^{\circ}$ direction and arrives at RX antenna \#1 in the $180^{\circ}$ direction. As seen from Fig. 7(a) and (c), antennas \#1 and \#4 have higher gain in the directions of $180^{\circ}$ and $0^{\circ}$, respectively. Thus, the direct wave is strongly received through channel $h_{14}$. This is why the CDF of $\left|h_{14}\right|$ is in the highest region and has the steepest gradient for $\mathrm{AS}=0.25 \lambda$ and $0.75 \lambda$. As for channel $h_{41}$ in the TX- $x / \mathrm{RX}-x$ orientation, the direct wave departs from $\mathrm{TX}$ antenna \#1 in the $0^{\circ}$ direction and arrives at RX antenna \#4 in the $180^{\circ}$ direction. Antennas \#1 and \#4 have lower gain in the directions of $0^{\circ}$ and $180^{\circ}$, respectively. The direct wave is weakly received through channel $h_{41}$. Thus, the CDF of $\left|h_{41}\right|$ is in the lowest region for $\mathrm{AS}=0.25 \lambda$ and $0.75 \lambda$. The relationship between the LOS component and antenna gain indicates that these two particular cases caused very different distributions among channel elements. Consequently, while it is well known that in LOS environments fading channels do not have independence due to the LOS component, the MIMO channel elements under the LOS condition do not have the same statistical characteristics.

\section{CDFs of Channel Capacities}

The channel capacity has been extensively used for evaluating the MIMO channel [7]-[10], [14]-[25]. This is the limit of digital communications that could only be achieved if we employed an ideal communication method (coding and modulation). As for the measured MIMO channel, the following equation gives the channel capacity of spatial multiplexing when channel state information is available only at the RX side

$$
\begin{aligned}
C & =\log _{2}\left\{\operatorname{det}\left(\boldsymbol{I}+\frac{1}{N_{T}} \frac{P_{\text {total }}}{P_{\mathrm{AEC}}} \boldsymbol{H}^{H} \boldsymbol{H}\right)\right\} \\
& =\sum_{i=1}^{N_{T}} \log _{2}\left(1+\frac{1}{N_{T}} \frac{P_{\text {total }}}{P_{\mathrm{AEC}}} \lambda_{i}\right) .
\end{aligned}
$$

Here, $\operatorname{det}(\cdot)$ denotes a determinant and $P_{\text {total }}$ indicates total TX power. $\lambda_{i}\left(i=1, \ldots, N_{T}\right)$ are eigenvalues obtained by eigenvalue decomposition of $\boldsymbol{H}^{H} \boldsymbol{H}$. $P_{\mathrm{AEC}}$ is TX power when the aforementioned SISO measurement in the AEC gives an average received $E_{s} / N_{0}$ of $0 \mathrm{~dB}$. Note that $P_{\text {total }} / P_{\mathrm{AEC}}$ in the above equation represents the normalized total TX power, which is used to evaluate the channel capacities and bit error rates

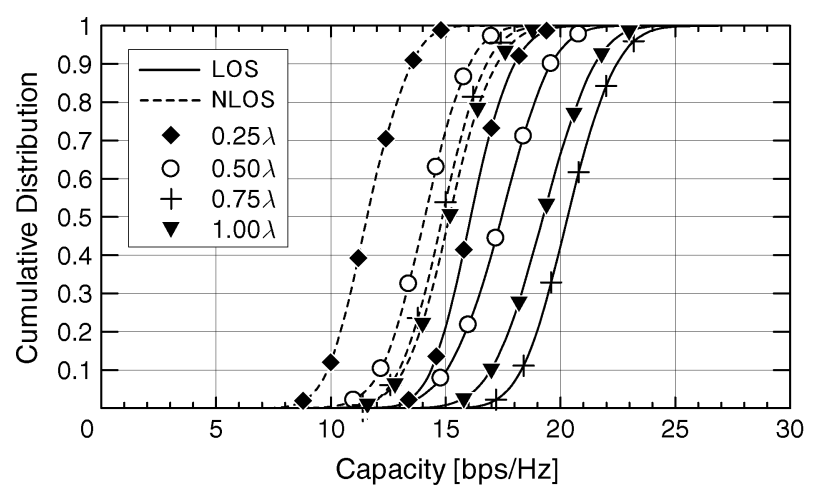

(a)

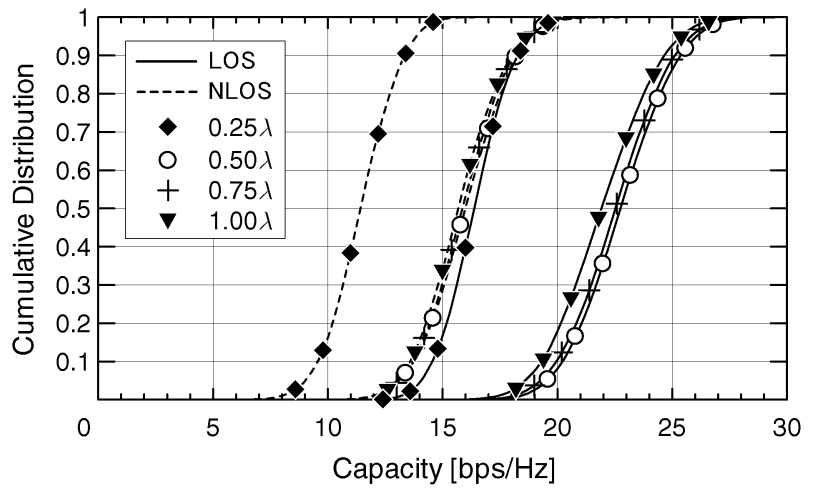

(b)

Fig. 10. CDFs of the measured $4 \times 4$ MIMO channel capacities for normalized total TX power of $20 \mathrm{~dB}$. (a) TX- $x / \mathrm{RX}-x$. (b) TX- $y / \mathrm{RX}-y$.

hereinafter. Therefore, we can compare performances under the same total TX power condition. Using the above equation, we examined CDFs of the $4 \times 4$ MIMO channel capacities for a normalized total TX power of $20 \mathrm{~dB}$.

The results shown in Fig. 10(a) and (b) are capacities for the TX- $x / \mathrm{RX}-x$ and TX- $y / \mathrm{RX}-y$ orientations, respectively. All of the capacities in the LOS condition are higher than those in the NLOS condition for both array orientations. The LOS component in the LOS condition enlarges the received power. Under a constant total TX power condition, the higher received power due to the LOS component improves the capacity. It is clear from (15) that the channel capacity is given by the eigenvalues of the MIMO channel. We can also say that the LOS component enlarges the maximum eigenvalue which significantly increases the channel capacity. Moreover, we can say that the LOS component enlarges the maximum eigenvalue and this larger eigenvalue causes such a high capacity. The eigenvalues will be discussed in detail in Section IV-F. On the other hand, the performance of $\mathrm{AS}=0.25 \lambda$ deteriorates in both LOS and NLOS conditions. This phenomenon can be analyzed by using the antenna patterns shown in Fig. 7. As described in Section IV-A, the patterns in the case of AS $=0.25 \lambda$ show serious gain decrease. We consider that this low antenna gain causes the decline in channel capacity. Except for the case of AS $=0.25 \lambda$, we see that in the LOS condition the TX- $y / \mathrm{RX}-y$ array orientation tends to give larger capacities than the TX- $x / \mathrm{RX}-x$ one. We illustrate this reason by using the antenna patterns shown in Fig. 7. As mentioned in Sections IV-A and IV-C, the antennas 
have higher gain than the single antenna in the $90^{\circ}$ direction especially in the cases of AS $=0.50 \lambda$ and $0.75 \lambda$. The direct signal is considered to be strongly received in the TX- $y / \mathrm{RX}-y$ orientation. This increases capacity. The reason that $\mathrm{AS}=1.00 \lambda$ gives a large capacity is conjectured to be lower fading correlations [Fig. 8(d)]. Moreover, in the LOS condition, the CDFs of the channel capacities strongly depend on the array configuration (antenna spacing and orientation).

There is an idea that an LOS channel can be modeled approximately as

$$
\boldsymbol{H}=\boldsymbol{H}_{\text {i.i.d. }}+\boldsymbol{H}_{\mathrm{LOS}}
$$

where $\boldsymbol{H}_{\text {i.i.d. }}$ is an $N_{R} \times N_{T}$ i.i.d. Rayleigh channel component matrix and $\boldsymbol{H}_{\mathrm{LOS}}$ is an $N_{R} \times N_{T}$ LOS component matrix [4]. Note that amplitudes of channel components are included in elements in these matrices. $\boldsymbol{H}_{\text {i.i.d. }}$ is a scattered ray component matrix in a multipath-rich environment with large antenna spacing. In this case, addition of $\boldsymbol{H}_{\mathrm{LOS}}$ to the scattered ray components leads to the large maximum eigenvalue and enhances the channel capacity. However, as stated in Section III, the scattered wave power in the LOS condition was $2.87 \mathrm{~dB}$ higher than that in the NLOS condition due to the short-delay paths caused by the direct wave. The direct LOS ray and enhanced scattered ray components further increase the channel capacity in the LOS environment. Also, when the received signal is linearly demultiplexed by a spatial filter, e.g., zero-forcing, the BER performance becomes better as the minimum eigenvalue becomes larger (see Sections IV-E and IV-F). It is not sure that the above model makes the minimum eigenvalue large, and it is difficult to arrive at the conclusion that spatial multiplexing in an LOS environment gives better BER performance. Therefore, we examined the behavior of spatial multiplexing in the LOS environment based on not channel models but measurement campaigns.

If we employ the LOS channel model given by (16), it is necessary to introduce the spherical-wave model [25] into $\boldsymbol{H}_{\mathrm{LOS}}$ in order to consider the variation of the LOS component phase because the distance between the TX and RX ends was relatively short. Here, let us consider a free space, i.e., $K=\infty$. In the case of $\mathrm{AS}=1.00 \lambda$ in the TX- $y / \mathrm{RX}-y$ array orientation, the fading correlation $\left|\rho_{14}\right|$ between antenna elements \#1 and \#4, which have the widest spacing of $3 \lambda$, is 0.95 . Under the SNR of $20 \mathrm{~dB}$, the channel capacity in this case becomes $30 \%$ higher than that in the case of $\operatorname{rank}(\boldsymbol{H})=1(\mathrm{TX}-x / \mathrm{RX}-x$ case in a free space). However, if an environment based on the channel model (16) has $K=1.66 \mathrm{~dB}$, which is the estimated Ricean factor in the measurement site, the correlation $\left|\rho_{14}\right|$ in the case of $A S=1.00 \lambda$ for the TX-y/RX- $y$ array orientation is 0.56 . Even for the TX- $x / \mathrm{RX}-x$ array orientation, the correlations for any antenna pairs are 0.59 . We confirmed that any array configurations give almost the same channel capacity and BER performance in the environment of $K=1.66 \mathrm{~dB}$. Consequently, we can say that the variation of the LOS component phase makes little impact on fading correlations and the performance of spatial multiplexing in propagation environments where the scattered wave power is comparable to the direct wave power. As
TABLE I

SimUlation PARAMETERS OF MIMO SPATIAL MULTIPLEXING

\begin{tabular}{c|c}
\hline \hline Array orientation & TX- $x /$ RX- $x$, TX- $y /$ RX- $y$ \\
\hline Total channel data & $7 \times 7 \times 1,601=78,449$ \\
\hline Modulation & QPSK \\
\hline Bit rate & $8\left(2 N_{T}\right)$ bits/symbol \\
\hline Burst length & 128 symbols (no coding) \\
\hline Thermal noise & White Gaussian noise \\
\hline RX processing & ZF spatial filtering \\
\hline \hline
\end{tabular}

aforementioned, the variation of the antenna gain due to mutual coupling changes the LOS component level, and this affects the performance in the LOS condition.

\section{E. $B E R$}

Earlier, we evaluated the performance of MIMO spatial multiplexing using the channel capacity. As aforementioned, the channel capacity obtained by (14) is the limit of the spatial multiplexing transmission. From the implementational viewpoint, we consider that evaluating the BER performance will be more practical. Thus, we conducted computer simulations of spatial multiplexing by using measured channel data and examined average BER performance under a constant bit rate requirement.

Table I lists the simulation parameters. As stated before, we had obtained 78449 channel data for each MIMO configuration, and we averaged all BERs for these channel data. We assumed that an independent QPSK-modulated uncoded stream was transmitted from each TX antenna with equal power. Therefore, $2 N_{T}$ bits/symbol were constantly transmitted. We employed a spatial filter based on a zero-forcing (ZF) scheme to detect the streams at the RX side [6]. This scheme suppresses interference completely by using the $\mathrm{ZF}$ weight matrix given by the following equation

$$
\boldsymbol{W}_{\mathrm{ZF}}=\left(\boldsymbol{H}^{H} \boldsymbol{H}\right)^{-1} \boldsymbol{H}^{H}
$$

An $N_{T}$-dimensional ZF output vector $\hat{\boldsymbol{s}}(t)$ is obtained when the RX signal vector $\boldsymbol{r}(t)$ is multiplied by $W_{\mathrm{ZF}}$ as follows:

$$
\begin{aligned}
\hat{\boldsymbol{s}}(t) & =\boldsymbol{W}_{\mathrm{ZF}} \boldsymbol{r}(t) \\
& =\boldsymbol{W}_{\mathrm{ZF}} \boldsymbol{H} \boldsymbol{s}(t)+\boldsymbol{W}_{\mathrm{ZF}} \boldsymbol{n}(t) \\
& =\boldsymbol{s}(t)+\boldsymbol{W}_{\mathrm{ZF}} \boldsymbol{n}(t) .
\end{aligned}
$$

Ordered successive detection such as BLAST [6], [7] was not applied. Results obtained by this processing show the performance of the simplest MIMO system without any error correction codes. This simulation also assumed that the RX side has perfect channel state information.

Fig. 11 shows the average BER performance of $4 \times 4$ MIMO spatial multiplexing. Fig. 11(a) and (b) are graphs for the TX- $x$ $/ \mathrm{RX}-x$ and TX- $y / \mathrm{RX}-y$ orientations, respectively. Because of $N_{T}=4$, the bit rate is 8 bits/symbol. The abscissa is normalized total TX power. We confirmed that the LOS condition gave higher correlations than the NLOS one (Fig. 8). However, Fig. 11 clearly shows that the BER performance for the LOS condition is better than that for the NLOS one. As mentioned in 


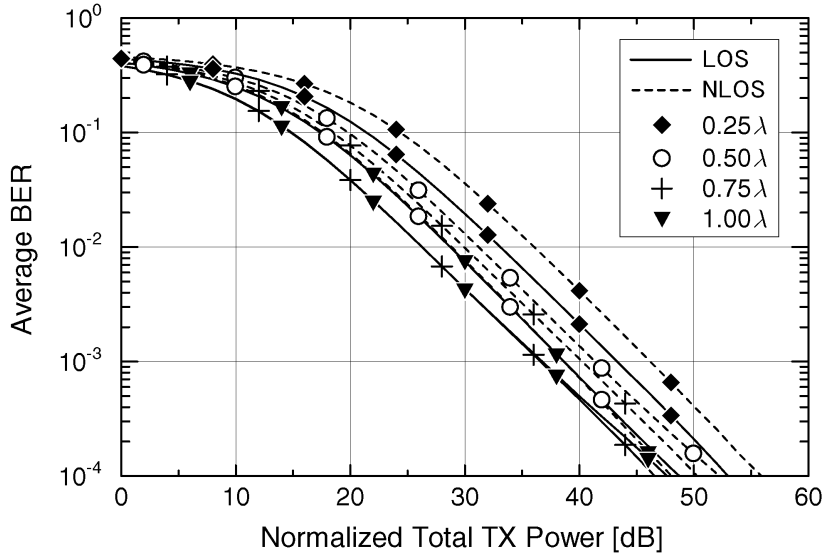

(a)

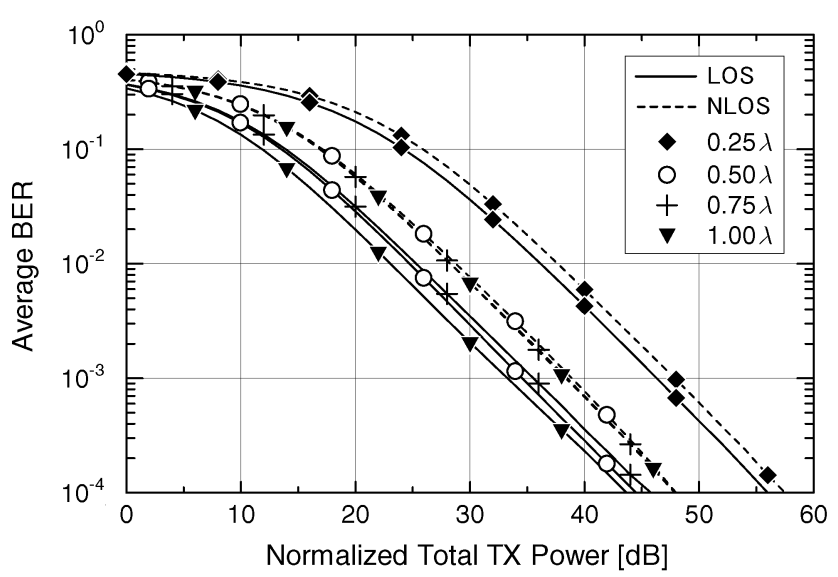

(b)

Fig. 11. Average BER performance of ZF processing for the measured $4 \times 4$ MIMO channels. (a) TX- $x / \mathrm{RX}-x$. (b) TX- $y / \mathrm{RX}-y$.

Section IV-D, the higher received power level given by the LOS component improves the BER performance.

Channel capacities shown in Fig. 10 indicate that all the CDFs under the LOS condition are better than those under the NLOS one regardless of the array orientation. In contrast, not all of the BERs under the LOS condition show better performance than those under the NLOS one because the cases of AS $=0.25 \lambda$ seriously deteriorate in both conditions. As stated in Section IV-C, the gain decrease of the inner two antenna elements \#2 and \#3 is especially noticeable in the patterns of the $\mathrm{AS}=0.25 \lambda$ case shown in Fig. 7(a). When we used this antenna array as the TX, more bit errors occurred on the streams from TX antennas \#2 and \#3. Average BER was strongly affected by such deteriorated streams.

The channel distributions shown in Fig. 9 indicate that the NLOS condition generally gives the linear parts of CDFs an increase of one order of magnitude with an amplitude increment of $10 \mathrm{~dB}$, and also that the LOS case has steeper curves owing to the direct wave. However, all the BER curves including those for the LOS condition show almost Rayleigh fading with first-order diversity in the high power region. That is, the BER is reduced by an order of magnitude when the TX power increases by 10 $\mathrm{dB}$. This behavior will be explained in the next subsection by using the minimum eigenvalue distributions.

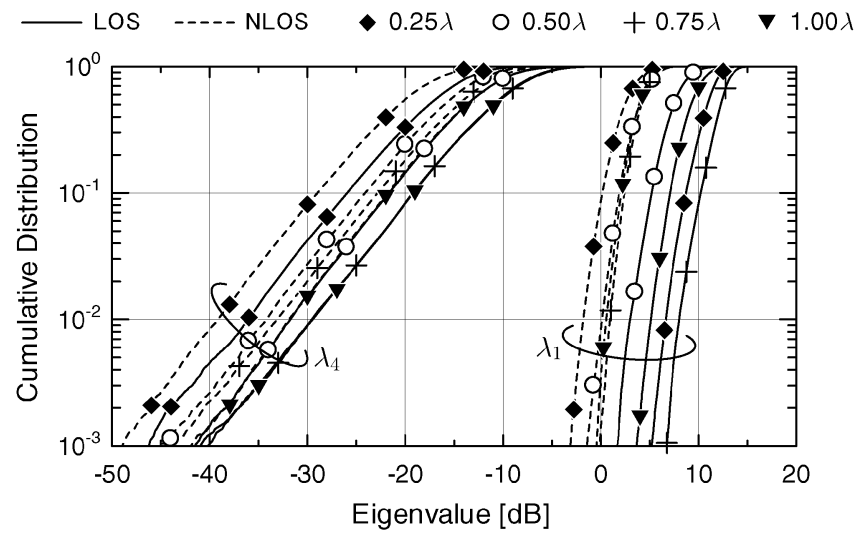

(a)

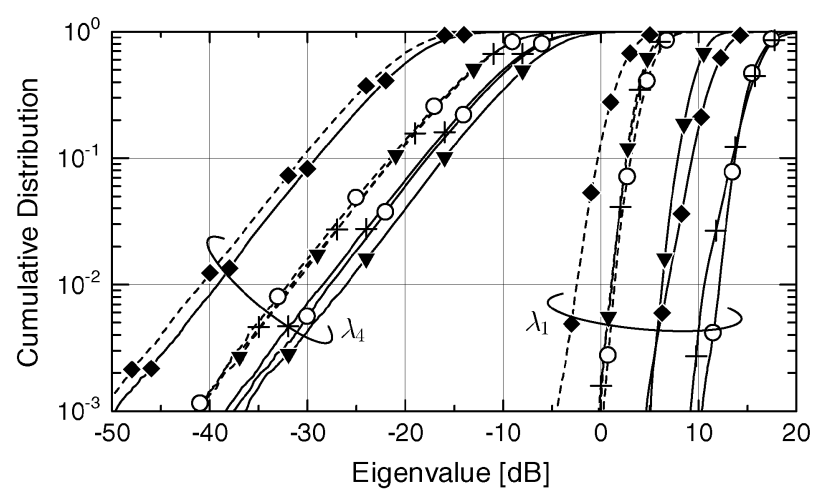

(b)

Fig. 12. CDFs of eigenvalues of $\boldsymbol{H}^{H} \boldsymbol{H}$ for the measured $4 \times 4$ MIMO channels. (a) TX- $x / \mathrm{RX}-x$. (b) TX-y/RX- $y$.

Although significant degradation occurs in the case of AS = $0.25 \lambda$ for both array orientations, under the LOS condition the antenna spacing and array orientation affect performance more than in the NLOS case. This is almost the same as the CDFs of capacities stated in Section IV-D.

\section{F. CDFs of Eigenvalues}

An $N_{T} \times N_{T}$ hermitian matrix $\boldsymbol{H}^{H} \boldsymbol{H}$ has $N_{T}$ nonnegative eigenvalues. We represent them in descending order $\lambda_{1}, \ldots, \lambda_{N_{T}}$. The number of positive eigenvalues equals the number of orthogonal channels between the TX and RX ends, and the eigenvalue is in proportion to the SNR of the channel. In other words, we can increase the transmission speed if the number of large eigenvalues increases. From (15), it is evident that a channel capacity is determined by the eigenvalues of the matrix $\boldsymbol{H}^{H} \boldsymbol{H}$. In particular, it is easy to imagine that the maximum eigenvalue $\lambda_{1}$ has the largest influence on the MIMO capacity. On the other hand, the ZF algorithm gives a tendency that the SNR at the RX end decreases as the minimum eigenvalue $\lambda_{N_{T}}$ decreases. This degrades the BER performance. Accordingly, we examined CDFs of eigenvalues of $\boldsymbol{H}^{H} \boldsymbol{H}$ in each MIMO configuration.

The CDFs of the eigenvalues are shown in Fig. 12. As well as Figs. 10 and 11, Fig. 11(a) and (b) are for the TX- $x / \mathrm{RX}-x$ and TX- $y / \mathrm{RX}-y$ array orientations, respectively. Although a $4 \times 4$ MIMO channel has four nonnegative eigenvalues, we show the 
CDFs of the maximum eigenvalues $\lambda_{1}$ and minimum eigenvalues $\lambda_{4}$ only. Compared with the CDFs of channel capacities shown in Fig. 10, we see that the order of CDFs of $\lambda_{1}$ does not necessarily correspond to that of the capacity distributions. This disagreement seems to be due to the effect of $\lambda_{2}$ and $\lambda_{3}$, which are not shown in Fig. 10 (see [26, Figs. 8, 9]). However, comparing the CDFs of the minimum eigenvalues $\lambda_{4}$ and average BERs shown in Fig. 11, we see that the orders are the same. Moreover, in the linear parts of the CDFs of $\lambda_{4}$, almost all of the values increase by about an order of magnitude with the increment of $10 \mathrm{~dB}$. The BER performance and its gradient, in short, seem to be dominated by the CDF of the minimum eigenvalue when only the ZF spatial filter is employed. We confirmed that channel capacity and BER are seriously degraded in the case of $\mathrm{AS}=0.25 \lambda$. The CDFs of the eigenvalues for AS $=0.25 \lambda$ tend to be in lower regions compared with the other cases. In particular, the minimum eigenvalues stay in the lower regions independently of the environmental conditions. This phenomenon causes the degradation of the MIMO performance.

As aforementioned, for the TX- $y / \mathrm{RX}-y$ orientation, the antennas have high gain in the LOS direction $\left(90^{\circ}\right.$ direction in Fig. 7) especially in the cases of AS $=0.50 \lambda$ and $0.75 \lambda$. The CDFs of the maximum eigenvalues $\lambda_{1}$ in these cases prove to have large values. Thus we can say that they are significantly affected by the direct wave.

\section{CONCLUSION}

We have evaluated channel capacities and average bit error rates of $4 \times 4$ MIMO spatial multiplexing by using measured MIMO channel data in an indoor multipath environment for the 5.2-GHz frequency band. The LOS and NLOS conditions for various MIMO configurations were analyzed in terms of the antenna patterns, fading correlations, CDFs of channel elements, and CDFs of eigenvalues.

It is well known that an LOS environment does not produce independent fading because an LOS component causes high fading correlation. From the measurement results, we confirmed this fact and also found that the LOS condition is not an identically distributed fading environment when each channel element is observed by antennas including mutual coupling effects. However, MIMO spatial multiplexing in the LOS environment provides higher capacities and lower bit error rates than in the NLOS environment under the same TX power condition. Hence, the utility of MIMO spatial multiplexing in an LOS environment has been proved. Meanwhile, the antenna gain in an array with narrow antenna spacing deteriorates because of mutual coupling effects. The decrease in gain will cause performance degradations in MIMO systems with numerous antennas. Under the LOS condition, the performance of MIMO spatial multiplexing strongly depends on the MIMO configuration.

This paper has presented the performance of $4 \times 4$ MIMO spatial multiplexing in a measurement site. We also conducted $2 \times 2$ MIMO measurement campaigns in the same and different indoor environments [26]-[28]. These measurement campaigns yielded almost the same conclusions as the ones presented in this paper. However, our measurement setups in these two environments were not practical but artificial, e.g., a large metal partition for the NLOS conditions and symmetric placement of TX and RX tables. Therefore, to confirm the tendencies shown in this paper, we should carry out more practical measurement campaigns assuming actual wireless systems such as wireless LANs for our future works.

\section{REFERENCES}

[1] G. J. Foschini and M. J. Gans, "On limits of wireless communications in a fading environment when using multiple antennas," Wireless Pers. Commun., pp. 311-335, Mar. 1998.

[2] I. E. Telatar, "Capacity of multi-antenna Gaussian channels," Euro. Trans. Telecommun., vol. 1, no. 6, pp. 585-595, Nov./Dec. 1999.

[3] D. Gesbert, M. Shafi, D. S. Shiu, P. Smith, and A. Naguib, "From theory to practice: An overview of MIMO space-time coded wireless systems," IEEE J. Sel. Areas Commun., vol. 21, no. 2, pp. 281-302, Apr. 2003.

[4] A. Paulraj, R. Nabar, and D. Gore, Introduction of Space-Time Wireless Communications. Cambridge, U.K.: Cambridge Univ. Press, 2003.

[5] A. J. Paulraj, D. A. Gore, R. U. Nabar, and H. Bölcskei, "An overview of MIMO communications-A key to gigabit wireless," Proc. IEEE, vol. 92, no. 2, pp. 198-218, Feb. 2004.

[6] G. D. Golden, G. J. Foschini, R. A. Valenzuela, and P. W. Wolniansky, "Detection algorithm and initial laboratory results using V-BLAST space-time communication architecture," Electron. Lett., vol. 35, no. 1, pp. 14-16, Jan. 1999.

[7] G. J. Foschini, G. D. Golden, R. A. Valenzuela, and P. W. Wolniansky, "Simplified processing for high spectral efficiency wireless communication employing multi-element arrays," IEEE J. Sel. Areas Commun., vol. 17, no. 11, pp. 1841-1852, Nov. 1999.

[8] G. J. Foschini, D. Chizhik, M. J. Gans, C. Papadias, and R. A. Valenzuela, "Analysis and performance of some basic space-time architectures," IEEE J. Sel. Areas Commun., vol. 21, no. 3, pp. 303-320, Apr. 2003.

[9] M. A. Jensen and J. W. Wallace, "A review of antennas and propagation for MIMO wireless communications," IEEE Trans. Antennas Propag., vol. 52, no. 11, pp. 2810-2824, Nov. 2004.

[10] D.-S. Shiu, G. J. Foschini, M. J. Gans, and J. M. Kahn, "Fading correlation and its effect on the capacity of multielement antenna systems," IEEE Trans. Commun., vol. 48, no. 3, pp. 502-513, Mar. 2000.

[11] I. J. Gupta and A. A. Ksienski, "Effect of mutual coupling on the performance of adaptive arrays," IEEE Trans. Antennas Propag., vol. AP-31, no. 5, pp. 785-791, Sep. 1983

[12] H. Iura, H. Yamada, Y. Ogawa, and Y. Yamaguchi, "Optimal antenna matching and mutual coupling effect of antenna array in MIMO receiver," IEICE Trans. Commun., vol. E90-B, no. 4, pp. 960-967, Apr. 2007.

[13] H. Steyskal and J. S. Herd, "Mutual coupling compensation in small array antennas," IEEE Trans. Antennas Propag., vol. 38, no. 12, pp. 1971-1975, Dec. 1990.

[14] P. N. Fletcher, M. Dean, and A. R. Nix, "Mutual coupling in multielement array antennas and its influence on MIMO channel capacity," Electron. Lett., vol. 39, no. 4, pp. 342-344, Feb. 2003.

[15] P. Uthansakul, M. Uthansakul, and M. E. Bialkowski, "Improving MIMO system capacity by compensating mutual coupling in transmitting/receiving array antennas," in Proc. IEEE Antennas Propag. Soc. Int. Symp., Jun. 2004, vol. 2, pp. 1724-1727.

[16] J. W. Wallace and M. A. Jensen, "Mutual coupling in MIMO wireless systems: A rigorous network theory analysis," IEEE Trans. Wireless Commun., vol. 3, no. 4, pp. 1317-1325, Jul. 2004.

[17] A. F. Molisch, M. Steinbauer, M. Toeltsch, E. Bonek, and R. S. Thomä, "Capacity of MIMO systems based on measured wireless channels," IEEE J. Sel. Areas Commun., vol. 20, no. 3, pp. 561-569, Apr. 2002.

[18] H. Özcelik, M. Herdin, H. Hofstetter, and E. Bonek, "Capacity of different MIMO systems based on indoor measurements at $5.2 \mathrm{GHz}$," in Inst. Elect. Eng. Europ. Pers. Mobile Commun. Conf. (EPMCC 2003), Apr. 2003, pp. 463-466.

[19] T. Mitsui, M. Otani, C. H. Y. Eugene, K. Sakaguchi, and K. Araki, "Indoor MIMO channel measurements for evaluation of effectiveness of array antenna configurations," Proc. IEEE VTC 2003-Fall, vol. 1, pp. 84-88, Oct. 2003.

[20] P. Kyritsi, D. C. Cox, R. A. Valenzuela, and P. W. Wolniansky, "Correlation analysis based on MIMO channel measurements in an indoor environment," IEEE J. Sel. Areas Commun., vol. 21, no. 5, pp. 713-720, Jun. 2003. 
[21] K. Yu, M. Bengtsson, B. Ottersten, D. McNamara, P. Karlsson, and M. Beach, "Modeling of wide-band MIMO radio channels based on NLOS indoor measurements," IEEE Trans. Veh. Technol., vol. 53, no. 3, pp. 655-665, May 2004.

[22] C. Waldschmidt and W. Wiesbeck, "Compact wide-band multimode antennas for MIMO and diversity," IEEE Trans. Antennas Propag., vol. 52, no. 8, pp. 1963-1969, Aug. 2004.

[23] D. P. McNamara, M. A. Beach, P. N. Fletcher, and P. Karlsson, "Capacity variation of indoor multiple-input multiple-output channels," Electron. Lett., vol. 36, no. 24, pp. 2037-2038, Nov. 2000.

[24] T. Svantesson and J. Wallace, "On signal strength and multipath richness in multi-input multi-output systems," in Proc. IEEE Int. Conf. Commun. (ICC'03), May 2003, vol. 4, pp. 2683-2687.

[25] J.-S. Jiang and M. A. Ingram, "Spherical-wave model for short-range MIMO," IEEE Trans. Commun., vol. 53, no. 9, pp. 1534-1541, Sep. 2005.

[26] Y. Ogawa, H. Nishimoto, T. Nishimura, and T. Ohgane, "Performance of MIMO spatial multiplexing in indoor line-of-sight environments," Proc. IEEE VTC2005-Fall, vol. 4, pp. 2398-2402, Sep. 2005.

[27] Y. Ogawa, H. Nishimoto, T. Nishimura, and T. Ohgane, "Performance of $2 \times 2$ MIMO spatial multiplexing in indoor environments," in 2005 IEEE/ACES Int. Conf. Wireless Commun. Appl. Computat. Electromagn., Apr. 2005, pp. 486-489.

[28] H. Nishimoto, Y. Ogawa, T. Nishimura, and T. Ohgane, "Availability of MIMO spatial multiplexing in line-of-sight channels," Proc. IEEE VTS APWCS2005, pp. 40-44, Aug. 2005.

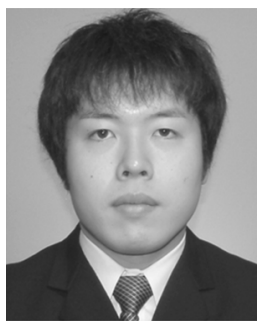

Hiroshi Nishimoto (S'05) received the B.E. and M.E. degrees from Hokkaido University, Sapporo, Japan, in 2003 and 2005, respectively.

$\mathrm{He}$ is currently working toward the Ph.D. degree at the Graduate School of Information Science and Technology, Hokkaido University. His research interests are in MIMO propagation measurement and MIMO communication systems. He has been a Research Fellow of the Japan Society for the Promotion of Science since 2005.

Mr. Nishimoto received the IEEE VTS Japan Chapter Student Paper Award and the Young Researchers' Award of IEICE Japan, both in 2007.

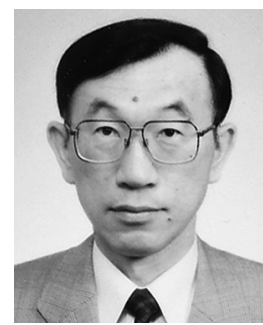

Yasutaka Ogawa (M'78-SM'90) received the B.E., M.E., and Ph.D. degrees from Hokkaido University, Sapporo, Japan, in 1973, 1975, and 1978, respectively.

Since 1979, he has been with Hokkaido University, where he is currently a Professor of the Graduate School of Information Science and Technology. During 1992-1993, he was with ElectroScience Laboratory, the Ohio State University, as a Visiting Scholar, on leave from Hokkaido University. His interests are in adaptive antennas, mobile communications, superresolution techniques, and MIMO systems.

Dr. Ogawa received the Young Researchers' Award of IEICE Japan in 1982 and the Best Paper Award from IEICE Japan in 2007.

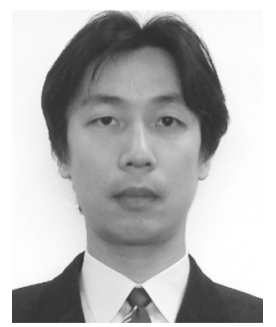

Toshihiko Nishimura (M'98) received the B.S. and M.S. degrees in physics and the Ph.D. degree in electronics engineering from Hokkaido University, Sapporo, Japan, in 1992, 1994, and 1997, respectively.

In 1998, he joined the Graduate School of Engineering (reorganized to Graduate School of Information Science and Technology) of Hokkaido University, where he is currently an Assistant Professor of the Graduate School of Information Science and Technology. His current research interests are in MIMO systems using smart antenna techniques.

Dr. Nishimura received the Young Researchers' Award of IEICE Japan in 2000 and the Best Paper Award from IEICE Japan in 2007.

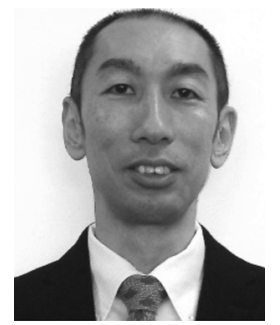

Takeo Ohgane (M'82) received the B.E., M.E., and $\mathrm{Ph} . \mathrm{D}$. degrees in electronics engineering from Hokkaido University, Sapporo, Japan, in 1984, 1986, and 1994, respectively.

From 1986 to 1992 , he was with the Communications Research Laboratory, Ministry of Posts and Telecommunications. From 1992 to 1995, he was on assignment at ATR Optical and Radio Communications Research Laboratory. Since 1995, he has been with Hokkaido University, where he is an Associate Professor. During 2005-2006, he was with the Centre for Communications Research, University of Bristol, U.K., as a Visiting Fellow. His interests are in MIMO signal processing for wireless communications.

Dr. Ohgane received the IEEE AP-S Tokyo Chapter Young Engineer Award in 1993, the Young Researchers' Award of IEICE Japan in 1990, and the Best Paper Award from IEICE Japan in 2007. 